\title{
A human-airway-on-a-chip for the rapid identification of candidate antiviral therapeutics and prophylactics
}

\author{
Longlong Si ${ }^{1,10}$, Haiqing Bai, ${ }^{1,0}$, Melissa Rodas ${ }^{1}$, Wuji Cao', Crystal Yuri Oh${ }^{1}$, Amanda Jiang ${ }^{1,2}$, \\ Rasmus Moller ${ }^{3}{ }^{3}$, Daisy Hoagland ${ }^{3}$, Kohei Oishi ${ }^{3}$, Shu Horiuchi ${ }^{3}$, Skyler Uhl ${ }^{3}$, Daniel Blanco-Melo ${ }^{3}$, \\ Randy A. Albrecht $\mathbb{B}^{3}$, Wen-Chun Liu ${ }^{3}$, Tristan Jordan ${ }^{3}$, Benjamin E. Nilsson-Payant $\mathbb{B}^{3}$, Ilona Golynker ${ }^{3}$, \\ Justin Frere $\mathbb{D}^{3}$, James Logue ${ }^{4}{ }^{4}$, Robert Haupt ${ }^{4}$, Marisa McGrath ${ }^{1}{ }^{4}$, Stuart Weston ${ }^{4}$, Tian Zhang ${ }^{5}$, \\ Roberto Plebani1,6, Mercy Soong ${ }^{1}$, Atiq Nurani1', Seong Min Kim¹, Danni Y. Zhu', Kambez H. Benam ${ }^{1,8,9}$, \\ Girija Goyal', Sarah E. Gilpin', Rachelle Prantil-Baun', Steven P. Gygi ${ }^{5}{ }^{5}$, Rani K. Powers', \\ Kenneth E. Carlson', Matthew Frieman ${ }^{(1)}{ }^{4}$, Benjamin R. tenOever ${ }^{3}{ }^{3}$ and Donald E. Ingber $\left(\mathbb{1}{ }^{1,2,7 凶}\right.$
}

The rapid repurposing of antivirals is particularly pressing during pandemics. However, rapid assays for assessing candidate drugs typically involve in vitro screens and cell lines that do not recapitulate human physiology at the tissue and organ levels. Here we show that a microfluidic bronchial-airway-on-a-chip lined by highly differentiated human bronchial-airway epithelium and pulmonary endothelium can model viral infection, strain-dependent virulence, cytokine production and the recruitment of circulating immune cells. In airway chips infected with influenza A, the co-administration of nafamostat with oseltamivir doubled the treatment-time window for oseltamivir. In chips infected with pseudotyped severe acute respiratory syndrome coronavirus 2 (SARS-CoV-2), clinically relevant doses of the antimalarial drug amodiaquine inhibited infection but clinical doses of hydroxychloroquine and other antiviral drugs that inhibit the entry of pseudotyped SARS-CoV-2 in cell lines under static conditions did not. We also show that amodiaquine showed substantial prophylactic and therapeutic activities in hamsters challenged with native SARS-CoV-2. The human airway-on-a-chip may accelerate the identification of therapeutics and prophylactics with repurposing potential.

$\mathrm{T}$ he increasing incidence of potential pandemic virusessuch as influenza A virus, Middle East respiratory syndrome coronavirus, severe acute respiratory syndrome coronavirus (SARS-CoV) and now SARS-CoV-2-requires development of new preclinical approaches that can accelerate the development of effective therapeutics and prophylactics. One of the most rapid ways to confront a pandemic challenge would be to repurpose existing drugs that are approved for other medical indications as antiviral therapeutics or prevention therapies. Although researchers and clinicians around the world are attempting to do this for the coronavirus disease 2019 (COVID-19) pandemic, the current approaches have been haphazard and generally rely entirely on the results of in vitro screens with cell lines. This has resulted in equivocal results regarding drug efficacies and possible toxicity risks, as in the case of hydroxychloroquine and chloroquine ${ }^{1-4}$; thus, there is a great need to address this problem in a more systematic and human-relevant way. Recognizing the potential danger of unforeseen pandemics over two years ago, the Defense Advanced Research Projects Agency (DARPA) and National Institutes of Health (NIH) funded work in our laboratory to explore whether human organ-on-a-chip (Organ Chip) microfluidic culture technology might be helpful in confronting potential biothreat challenges. We previously showed that Organ Chips can recapitulate human organ physiology, disease states and therapeutic responses to clinically relevant drug exposures with high fidelity $^{5-9}$. Here we show that human lung bronchial-airway chips (Airway Chips) may be used to model the human-lung responses to viral infection in vitro and, in concert with higher throughput cell-based assays and animal models, to identify existing approved drugs that have the potential to be repurposed for treating or preventing the spread of viral pandemics caused by influenza A or SARS-CoV-2 viruses.

Infections by respiratory viruses and antiviral drug screening assays are currently studied in vitro using established cell lines, primary tissue-derived human cells and human organoids, and ex vivo in human lung tissue cultures despite all having notable limitations (Supplementary Table 1$)^{10-13}$. For example, cell lines commonly demonstrate various defects in their capacity to mount an antiviral response because normally elicited interferons cause cell-cycle

'Wyss Institute for Biologically Inspired Engineering, Harvard University, Boston, MA, USA. ${ }^{2}$ Vascular Biology Program and Department of Surgery, Boston Children's Hospital and Harvard Medical School, Boston, MA, USA. ${ }^{3}$ Department of Microbiology, Icahn School of Medicine at Mount Sinai, New York, NY, USA. ${ }^{4}$ Department of Microbiology and Immunology, University of Maryland School of Medicine, Baltimore, MD, USA. ${ }^{5}$ Department of Cell Biology, Harvard Medical School, Boston, MA, USA. ${ }^{6}$ Center on Advanced Studies and Technology (CAST), Department of Medical, Oral and Biotechnological Sciences, "G. d'Annunzio" University of Chieti-Pescara, Chieti, Italy. ${ }^{7}$ Harvard John A. Paulson School of Engineering and Applied Sciences, Cambridge, MA, USA. ${ }^{8}$ Present address: Division of Pulmonary, Allergy and Critical Care Medicine, Department of Medicine, University of Pittsburgh, Pittsburgh, PA, USA. ${ }^{9}$ Present address: Department of Bioengineering, University of Pittsburgh, Pittsburgh, PA, USA. ${ }^{10}$ These authors contributed equally: Longlong Si, Haiqing Bai. 凶e-mail: don.ingber@wyss.harvard.edu 
arrest and so this dynamic is often selected against with continuous passaging ${ }^{14}$. Cell lines and even human primary lung epithelial cells cultured in conventional cultures also do not exhibit the highly differentiated tissue structures and functions (for example, ciliary differentiation and mucociliary clearance) seen in the living human lung. Explant cultures of human respiratory-tract tissue circumvent this limitation but their availability is limited and their viability can only be maintained for a short time ${ }^{12,15}$. Although human lung organoids provide a more functional lung epithelium, they do not allow culturing of the epithelium at an air-liquid interface (ALI) or modelling of other physiologically relevant organ-level features of the lung-such as, mucus layer formation, mucociliary clearance, cross-talk between the epithelium and endothelium, and recruitment of circulating immune cells $s^{10,11}$, all of which play key roles in the host responses to infection by respiratory viruses ${ }^{10,11,15}$. Moreover, in all of these culture systems and in Transwell co-culture models that support ALI formation, drug studies are carried out under static conditions that cannot predict human responses to clinically relevant, dynamic drug exposure profiles that result from complex pharmacokinetics (PK) in vivo ${ }^{16}$. Thus, there is an urgent need for alternative preclinical in vitro models that mimic human-lung responses to infection by potential pandemic respiratory viruses and-because of their ability to recapitulate human organ-level physiology, pathophysiology and clinically relevant drug exposures-human Airway Chips offer a potential solution.

\section{Results}

Influenza $A$ virus infection and immune responses replicated in human Airway Chips. To initially assess whether Organ Chip technology $y^{5-8}$ can be used to create a preclinical in vitro model for the identification of new potential treatment strategies for pandemic respiratory viruses, we initially tested it against a drug that is used clinically for the treatment of influenza A virus infections. The human Airway Chip is a microfluidic device that contains two parallel microchannels separated by an extracellular matrix-coated porous membrane (Fig. 1a) ${ }^{17}$. Primary human lung bronchial-airway basal stem cells are cultured under an ALI on one side of the membrane in the 'airway channel' while interfaced with a primary human lung endothelium cultured on the opposite side of the same membrane, which is exposed to continuous fluid flow of culture medium in the parallel 'vascular channel' (Fig. 1a). This device supports differentiation of the lung bronchial-airway basal stem cells into a mucociliated, pseudostratified bronchial-airway epithelium with proportions of airway-specific cell types (ciliated cells, mucus-producing goblet cells, club cells and basal cells) that are similar to those in the human airway (Fig. 1b and Supplementary Fig. 1a). This is also accompanied by the establishment of continuous ZO1-containing tight junctions and cilia (Fig. 1c), permeability barrier properties and mucus production (Supplementary Fig. 1b,c) similar to those observed in the human airway in vivo ${ }^{18}$ as well as in previous Airway Chip studies using a membrane with smaller pores that did not permit immune-cell transmigration ${ }^{17}$. The underlying human pulmonary microvascular endothelium also forms a continuous planar cell monolayer with cells linked by VE-cadherin-containing adherens junctions (Fig. 1c) as it does in vivo.

Importantly, the highly differentiated airway epithelium in the Airway Chip expresses higher levels of genes encoding multiple serine proteases involved in viral entry-including TMPRSS2, TMPRSS4, TMPRSS11D and TMPRSS11E(DESC1)_compared with established cell lines (for example, MDCK cells) that are often used to study influenza virus infection in vitro (Supplementary Fig. 1d); these proteases are essential for the activation and propagation of influenza viruses in vivo ${ }^{12}$. In addition, compared with their initial state following seeding, differentiation of the airway epithelial cells at an ALI on-chip is accompanied by large increases in the levels of expression of messenger RNA (mRNA) of the TMPRSS2 protease (Fig. 1d) and similar increases in the levels of angiotensin converting enzyme-2 (ACE2) mRNA (Fig. 1e) and protein (Fig. 1f) are also observed. ACE2 plays a central role in lung physiology and pathophysiology ${ }^{19}$ in addition to serving as the main receptor for SARS-CoV-2, which mediates its infection ${ }^{20,21}$.

When green fluorescent protein (GFP)-expressing influenza A/PuertoRico8/34 (H1N1) virus was introduced into the air channel of the microfluidic chip to mimic in vivo infection with airborne virus (Fig. 1a), real-time fluorescence microscopy analysis confirmed that the influenza A virus infected the human airway epithelial cells (Fig. 1c and Supplementary Videos 1,2) and this was accompanied by damage to the epithelium, including disruption of tight junctions, loss of apical cilia (Fig. 1c) and compromised barrier function (Fig. 1g). Significantly less infection was detected in undifferentiated airway basal epithelium previous to culture at an ALI on-chip and there was no detectable direct infection of the endothelium by the virus (Supplementary Fig. 2a). Interestingly, however, infection with influenza A virus led to the disruption of the lung endothelium in the Airway Chip, as evidenced by a loss of VE-cadherin-containing adherens junctions (Fig. 1c), which is consistent with the vascular leakage that is induced in the lungs of human patients with influenza ${ }^{22}$.

Analysis of the replication kinetics of six different influenza A virus strains-including clinical isolates (A/Netherlands/602/2009 (NL/09; H1N1), A/Hong Kong/8/68 (HK/68; H3N2), A/ Panama/2007/99 (Pan/99; H3N2) and A/Hong Kong/156/1997 (HK/97; H5N1)) and cell-culture strains (influenza Puerto Rico/8/34 (H1N1) and A/WSN/1933 (WSN; H1N1)) - showed that all H1N1 and $\mathrm{H} 3 \mathrm{~N} 2$ virus variants propagated efficiently as demonstrated by large $\left(1 \times 10^{3}-1 \times 10^{4}\right.$-fold $)$ increases in the viral titres over $24-48 \mathrm{~h}$ in highly differentiated human lung airway epithelium on-chip (Fig. 2a). Although the H5N1 virus increased more than tenfold in number, it grew significantly slower (Fig. 2a), which is consistent with clinical observations that $\mathrm{H} 5 \mathrm{~N} 1$ predominantly infects the lower respiratory tract (that is, rather than the large airway) ${ }^{23}$. Notably, the H3N2 virus strains (HK/68 and Pan/99) exhibited an approximately tenfold-higher replication efficiency than the H1N1 strains (PR8, WSN and NL/09; Fig. 2a) and caused significantly more severe barrier disruption (Fig. $1 \mathrm{~g}$ ) as well as a higher cilia loss (Supplementary Fig. 2b). These results suggest that these H3N2 virus strains are more infectious than $\mathrm{H} 1 \mathrm{~N} 1$ virus strains, which could potentially contribute, at least in part, to the increased clinical severity of the H3N2 influenza infections observed in humans ${ }^{24}$. Donor-to-donor variability in terms of sensitivity to infection with influenza virus was minimal in these studies, as similar viral infectivity was obtained in chips derived from five different healthy epithelial cell donors (Supplementary Fig. 2c).

Recruitment of circulating immune cells, such as neutrophils, under dynamic flow to the site of infection in the airway epithelium contributes greatly to the pathogenesis of influenza A virus in the lung ${ }^{25}$; however, this process has not been well investigated in existing in vitro models due to their two-dimensional static nature. When primary human neutrophils were perfused through the vascular channel of Airway Chips infected with $\mathrm{H} 1 \mathrm{~N} 1$ or $\mathrm{H} 3 \mathrm{~N} 2$ virus, we observed recruitment of these circulating immune cells to the apical surface of the activated lung endothelium within minutes, whereas minimal neutrophil adhesion was observed in uninfected chips (Fig. 2b, top, and Supplementary Videos 3,4). This was followed by transmigration of the neutrophils through the endothelium and the extracellular matrix-coated pores of the intervening membrane, and up into the airway epithelium over hours (Fig. 2b, bottom). The neutrophils targeted the nucleoprotein-positive airway cells infected with influenza A virus (Supplementary Fig. 3a) and induced them to coalesce into clusters that decreased in size over time. This resulted in clearance of the virus, as evidenced by the disappearance 
a

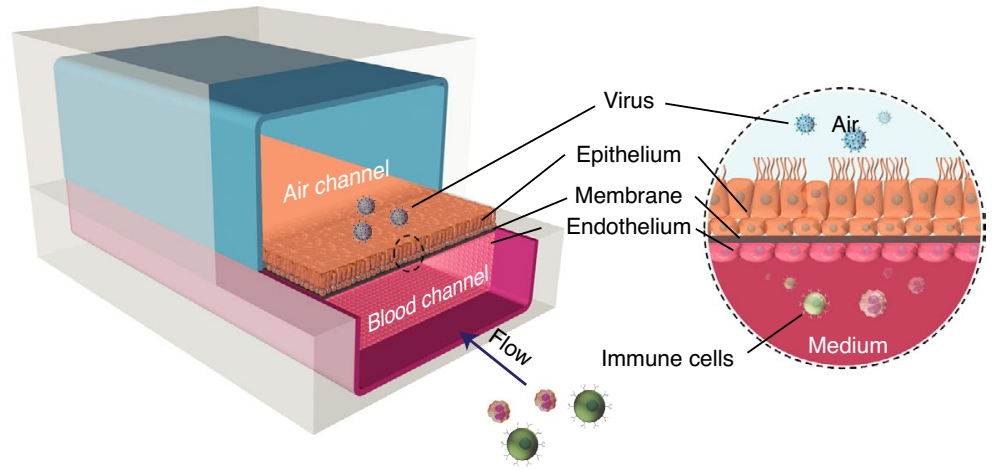

b
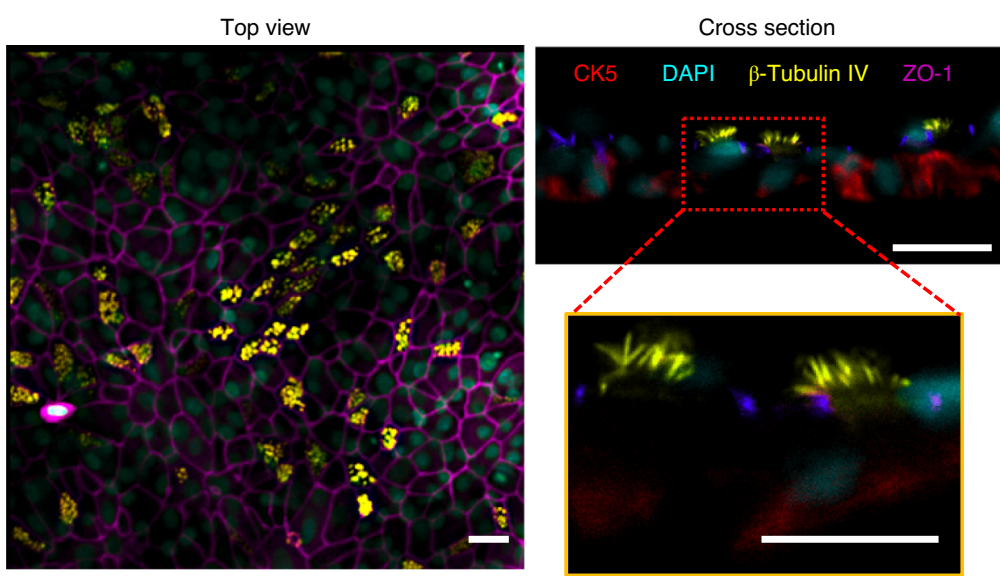

c

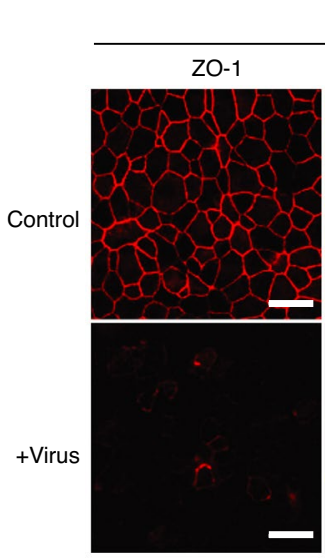

Epithelium Cilia

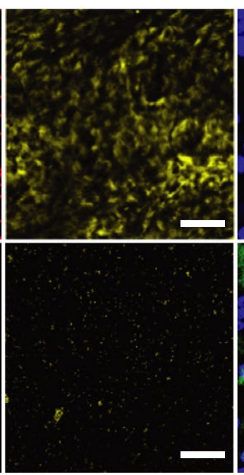

d

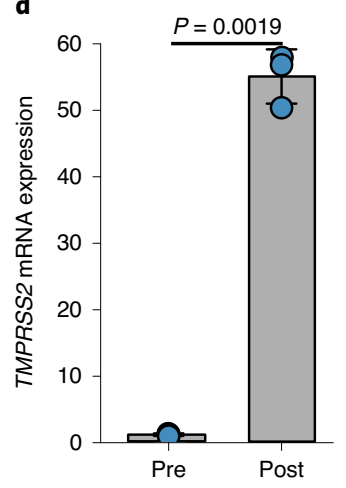

e

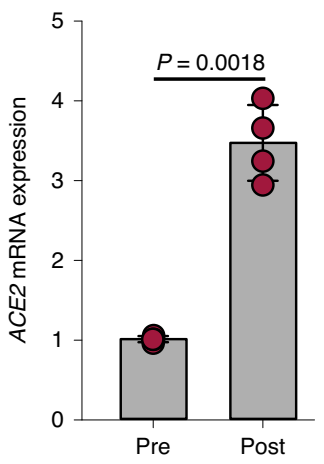

f

Pre

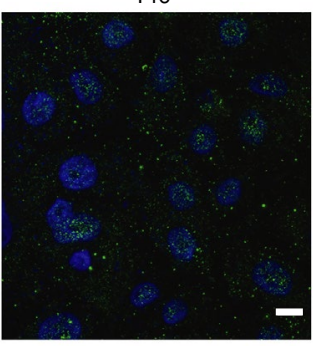

Post

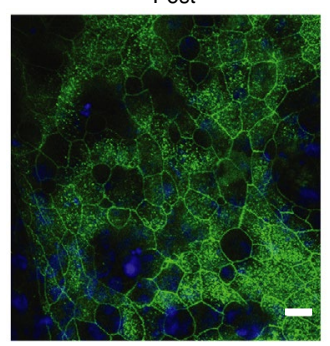

g

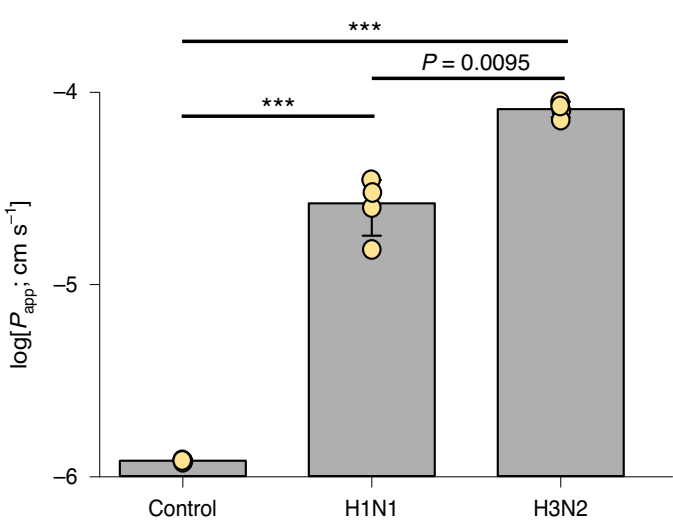

Fig. 1 | Characterization of the human Airway Chip and its infection with influenza virus. a, Schematic of a cross section through the Airway Chip. b. Immunofluorescence staining of the human Airway Chip reveals a pseudostratified epithelial layer containing CK $5^{+}$basal cells and $\beta$-tubulin IV ${ }^{+}$ciliated cells. DAPI, 4,6-diamidino-2-phenylindole. c, Immunofluorescence micrographs showing the distribution of ZO1-containing tight junctions and cilia in the epithelium and VE-cadherin-containing adherens junctions in the endothelium of the Airway Chip in the absence (Control; top) or presence (+virus; bottom) of infection with GFP-expressing influenza PR8 (H1N1) virus (MOI=0.1) for 48 h. Blue, DAPI-stained nuclei. d, Fold change in the mRNA levels of TMPRSS2 in the well-differentiated primary human airway epithelium on-chip compared with the same cells before differentiation. e, Fold change in the mRNA levels of ACE2 in the well-differentiated primary human airway epithelium on-chip compared with the same cells following differentiation. f, Immunofluorescence micrographs showing the expression of the ACE2 receptor in the well-differentiated primary human airway epithelium on-chip compared with the same cells before differentiation. Blue, DAPI-stained nuclei; green, ACE2. $\mathbf{g}$, Increase in barrier permeability, as measured by the apparent permeability $\left(\log \left[P_{\text {app }}\right]\right)$, in the human Airway Chip 48 h post infection with PR8 (H1N1) or HK/68 (H3N2) virus (MOI=0.1) compared with no infection (Control). Data represent the mean \pm s.d.; $n=4(\mathbf{e}, \mathbf{g})$ and 3 (f) biological chip replicates; ${ }^{\star \star \star} P<0.001$. Pre, before differentiation; post, post differentiation. Scale bars, $50 \mu \mathrm{m}$.

of $\mathrm{GFP}^{+}$cells over a period of 1-2 days, which did not occur in the absence of neutrophils (Fig. 2b, bottom). Consistent with the ability of $\mathrm{H} 3 \mathrm{~N} 2$ virus to induce stronger inflammation relative to H1N1 in vivo ${ }^{26}, \mathrm{H} 3 \mathrm{~N} 2$ also stimulated more neutrophil recruitment than
H1N1 (Fig. 2c) and neutrophil infiltration into the epithelium significantly decreased the viral titres of both H1N1 and H3N2 on-chip (Fig. 2d), consistent with the protective role that neutrophils provide by clearing virus in vivo ${ }^{25}$. H1N1 infection was also accompanied by 


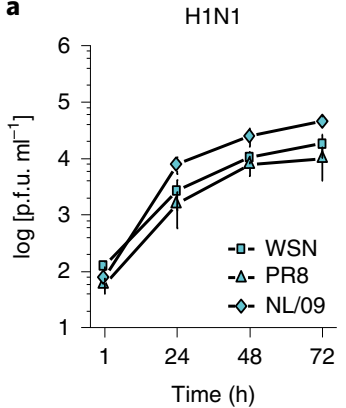

c

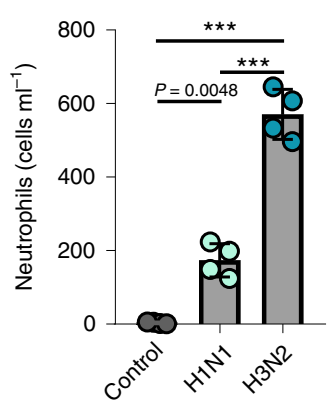

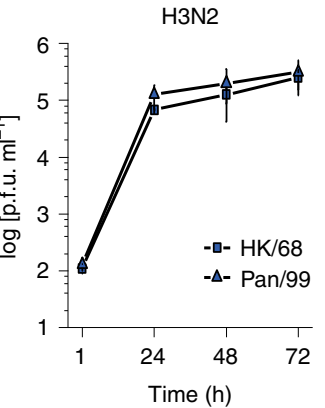

d

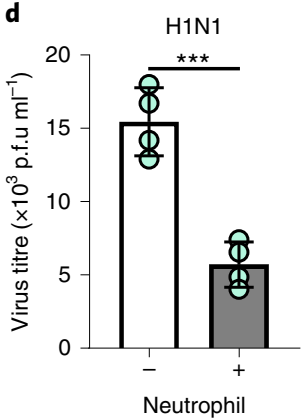

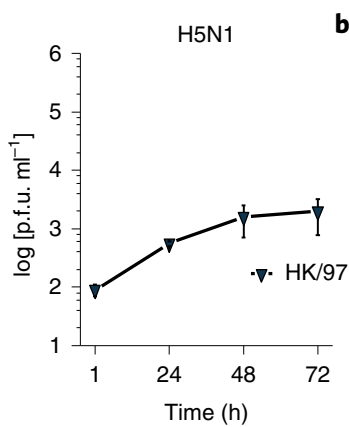

b Time (min)

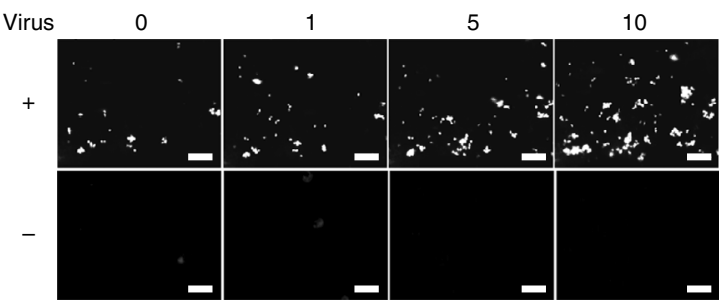

Time (h)

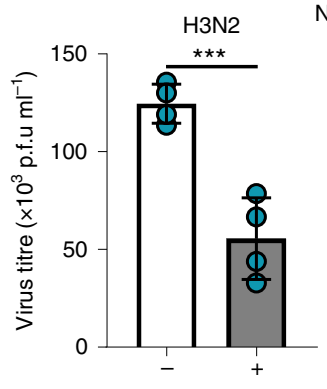

Neutrophil 0

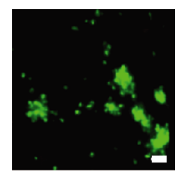

24

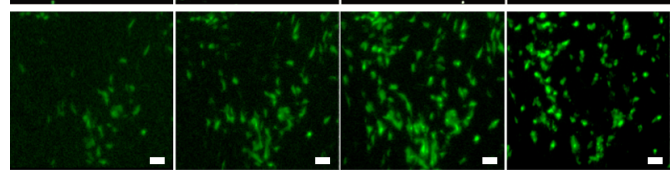

Neutrophi
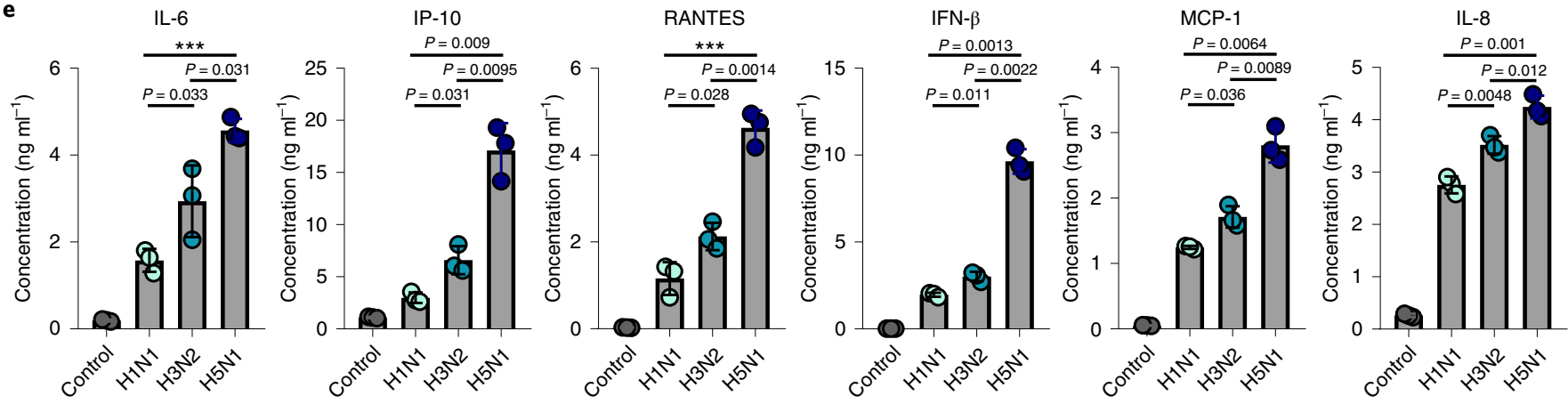

Fig. 2 | Infection with multiple influenza strains in human Airway Chips and the resulting immune responses. a, Replication kinetics of influenza H1N1 virus strains WSN, PR8 and NL/09 (left); influenza H3N2 virus strains HK/68 and Pan/99 (middle); and a H5N1 strain (right) following infection of human Airway Chips at $\mathrm{MOI}=0.001$. b, Neutrophil responses to influenza infection in the human Airway Chip. Sequential immunofluorescence micrographs showing time-dependent recruitment of neutrophils (white) to the apical surface of the endothelium (unlabelled) in a human Airway Chip infected with influenza PR8 (H1N1) virus (top); uninfected chips were used as controls. Immunofluorescence micrographs showing time-dependent recruitment of neutrophils (white) to the epithelium (unlabelled) and clearance of clustered epithelial cells infected with GFP-expressing PR8 (H1N1) virus (green; bottom); infected chips without neutrophils were used as controls. Scale bars, $50 \mu \mathrm{m}$. c, Numbers of neutrophils recruited to the epithelium in response to infection by $\mathrm{H} 1 \mathrm{~N} 1$ or $\mathrm{H} 3 \mathrm{~N} 2$, and the baseline level of neutrophils in uninfected chips (control). d, Virus titres of human Airway Chips infected with WSN (H1N1; left) or HK/68 (H3N2; right) in the presence (+) or absence (-) of added neutrophils. e, Production of the indicated cytokines and chemokines in the human Airway Chip at $48 \mathrm{~h}$ post infection ( $\mathrm{MOI}=0.1)$ with different clinically isolated influenza virus strains - that is, $\mathrm{NL} / 09$ ( $\mathrm{H} 1 \mathrm{N1})$, Pan/99 (H3N2) and HK/97 (H5N1). Data represent the mean \pm s.d.; $n=4$ (a,c,d) and 3 (e) biological chip replicates; ${ }^{\star \star \star} P<0.001$.

increased secretion of various inflammatory cytokines and chemokines-IL-6, IP-10, RANTES, interferon- $\beta$, MCP- 1 and IL-8-which could easily be measured in the effluent from the vascular channel (Fig. 2e). The added presence of neutrophils also further increased cytokine production and caused more damage to the tissue barrier in the human Airway Chip (Supplementary Fig. 3b,c).

Variations in secretion of proinflammatory mediators in the human lung airway contribute to differences in pathogenesis and morbidity observed for different influenza A virus strains, and the analysis of the levels of cytokines helps clinicians assess disease severity. We thus compared the innate immune responses of the human Airway Chip to infection with three patient-derived influenza A virus strains with differing virulence: NL/09 (H1N1), Pan/99 (H3N2) and $\mathrm{HK} / 97$ (H5N1). When the chips were infected with H3N2 and H5N1 viruses that are known to produce more severe clinical symptoms than H1N1 in patients, we found that they also stimulated the production of higher levels of cytokines and chemokines and the most virulent $\mathrm{H} 5 \mathrm{~N} 1$ strain induced the highest concentrations (Fig. 2e) despite exhibiting lower replication kinetics (Fig. 2a). These results mirror the clinical finding that aberrant host responses and a higher case fatality compared with $\mathrm{H} 1 \mathrm{~N} 1$ or $\mathrm{H} 3 \mathrm{~N} 2$ are characteristics of human infections with $\mathrm{H} 5 \mathrm{~N} 1$ and that patients infected with $\mathrm{H} 5 \mathrm{~N} 1$ have increased serum concentrations of these inflammatory factors, which play major roles in disease pathogenesis ${ }^{26}$.

Recapitulation of the effects of antiviral therapeutics used in the clinic. To explore whether the Airway Chip can be used to evaluate the efficacy of potential antiviral therapeutics, we first tested 

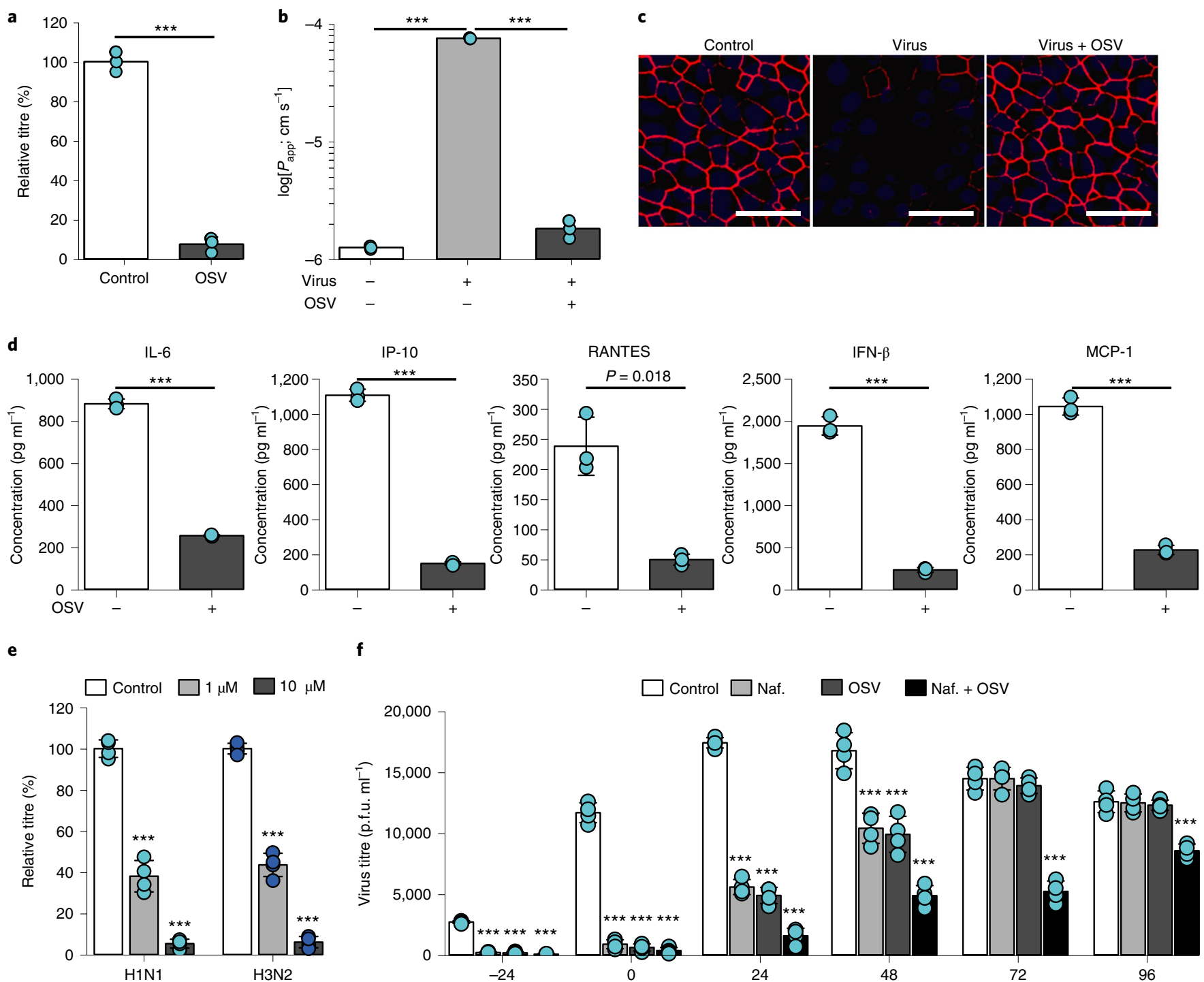

f

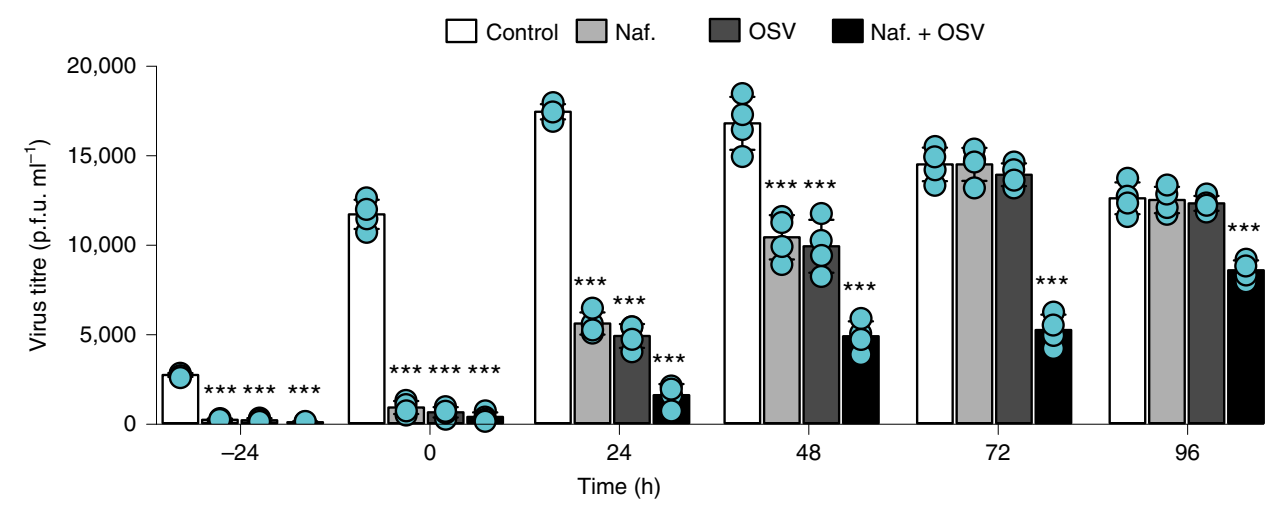

Fig. 3 | Effects of anti-influenza therapeutics in the human Airway Chip. a, Relative plaque titres of progeny virus in the absence (control) or presence of $1 \mu \mathrm{M}$ oseltamivir acid (OSV) $48 \mathrm{~h}$ post infection with WSN $(\mathrm{H} 1 \mathrm{N1}$; $\mathrm{MOI}=0.1)$. b. Barrier permeability $\left(\log \left[P_{\text {app }}\right]\right)$ measured under control conditions in the human Airway Chip (-virus) or $48 \mathrm{~h}$ post infection with WSN (+virus) with (+) or without (-) OSV. c, Immunofluorescence micrographs showing the distribution of ZO1-containing tight junctions in airway epithelium under baseline conditions (control) or infected with WSN alone or in the presence of OSV ( $48 \mathrm{~h}$ post infection). Scale bars, $50 \mu \mathrm{m}$. d, Production of cytokines in human Airway Chip $48 \mathrm{~h}$ post infection with WSN in the presence ( + ) or absence (-) of OSV. e, Effects of nafamostat, at concentrations of $1 \mu \mathrm{M}$ or $10 \mu \mathrm{M}$, on the virus replication of H1N1 and H3N2 in Airway Chips $48 \mathrm{~h}$ post infection compared with untreated chips (control). In a-e, the drugs were added at the same time that the chips were infected with virus. f, Therapeutic window. Effects of nafamostat (Naf.), OSV and their combination on viral titres when added to H1N1 virus-infected human Airway Chips at the indicated times (that is, $24 \mathrm{~h}$ before virus addition, at the time of infection, and $24,48,72$ and $96 \mathrm{~h}$ after infection). The effects on viral load were analysed at $48 \mathrm{~h}$ following the addition of the drugs. Note the synergistic effects of these two drugs at the later times. Data represent the mean \pm s.d.; $n=4$ (e,f) and 3

$(\mathbf{a}, \mathbf{b}, \mathbf{d})$ biological chip replicates; ${ }^{\star * \star} P<0.001$.

oseltamivir (Tamiflu), which is the anti-influenza drug that is most widely used in the clinic. As oseltamivir is metabolized by the liver to release oseltamivir acid in vivo, we introduced this active metabolite into the vascular channel of Airway Chip infected with H1N1 virus, mimicking its blood levels after oral administration. Oseltamivir $(1 \mu \mathrm{M})$ efficiently inhibited the replication of influenza A virus (Fig. 3a), prevented virus-induced compromise of barrier function (Fig. 3b) and disruption of epithelial tight junctions (Fig. 3c), and decreased the production of multiple cytokines and chemokines on-chip (Fig. 3d). Importantly, similar anti-influenza efficacy was detected in a randomized control trial where treatment with Oseltamivir also led to one order of magnitude drop in the viral titres in nasopharyngeal samples provided by 350 patients ${ }^{27}$. Thus, the Airway Chip faithfully replicates the effects of oseltamivir previously observed in humans, suggesting that it may serve as a useful preclinical model to evaluate potential therapies for virus-induced human lung infections in a preclinical setting.

Repurposing of approved drugs as potential anti-influenza therapeutics. Given that the host serine proteases on human airway epithelial cells play critical roles in the propagation of influenza A virus ${ }^{12,28}$, and their expression is significantly elevated in the differentiated Airway Chip (Fig. If and Supplementary Fig. 1d), we explored whether existing approved drugs that inhibit serine 
proteases could suppress infection by delivering them into the airway channel of influenza virus-infected chips (for example, to mimic intratracheal delivery by aerosol, nebuliser or inhaler). These studies revealed that two anticoagulant drugs used in the clinical setting, nafamostat (Fig. 3e) and Trasylol (Supplementary Fig. 4a), significantly reduced the titres of influenza $\mathrm{H} 1 \mathrm{~N} 1$ and $\mathrm{H} 3 \mathrm{~N} 2$ on-chip. Further exploration of the actions of nafamostat revealed that it protects the airway barrier function (Supplementary Fig. 4b) and tight-junction integrity (Supplementary Fig. 4c), and decreases the production of cytokines and chemokines (Supplementary Fig. 4d). Nafamostat and the other protease inhibitors seemed to act by efficiently blocking the enzymatic cleavage of the influenza A virus protein haemagglutinin (HA0) into the subunits HA1 and HA2 by the serine proteases TMPRSS11D and TMPRSS2 (Supplementary Fig. 4e), which is required for viral entry ${ }^{29}$.

When we added nafamostat or oseltamivir at different time points before and after influenza virus infection on-chip, both nafamostat and oseltamivir exhibited prophylactic and therapeutic effects (Fig. 3f). However, oseltamivir only produced therapeutic effects when it was administered within $48 \mathrm{~h}$ post infection on-chip (Fig. 3f). This is consistent with the observation that oseltamivir is only recommended for clinical use within 2 days of influenza virus infection $^{30}$, which is one of the important limitations of using this antiviral therapeutic clinically. Nafamostat also exhibited its inhibitory effects over a 48-h time period (Fig. 3f). Impressively, however, combined administration of nafamostat and oseltamivir exerted a more potent inhibition of influenza virus infection and this combined regimen was able to double the treatment-time window of oseltamivir from 48 to $96 \mathrm{~h}$ (Fig. 3f).

Identification of approved drugs as SARS-CoV-2 entry inhibitors. Having found that the Airway Chips faithfully recapitulate many clinical features of the human-lung responses to influenza infection, we quickly pivoted our effort to focus on SARS-CoV-2 infection when we learned of the emerging COVID-19 pandemic. To initiate work immediately in our biosafety-level (BSL)-2 laboratory and alleviate safety concerns, we designed SARS-CoV-2 pseudoparticles (SARS-CoV-2pp) that contain the SARS-CoV-2 spike $(\mathrm{S})$ protein assembled onto luciferase reporter gene-carrying retroviral core particles ${ }^{31}$, based on the genome sequence of SARS-CoV-2 released in GenBank on 12 January 2020 (ref. ${ }^{32}$ ). Pseudotyped S protein-expressing viral particles faithfully reflect key aspects of native SARS-CoV-2 entry into host cells via binding to its ACE2 receptor ${ }^{33}$ and they can thus be used to test potential entry inhibitors of SARS-CoV-2 (refs. ${ }^{20,31}$ ). Validation studies confirmed that the SARS-CoV-2 S protein integrated into the SARS-CoV-2pp, as indicated by western blotting (Supplementary Fig. 5a) and shown in other pseudotyped SARS-CoV-2 viruses ${ }^{20}$. We also observed efficient SARS-CoV-2pp infection in Huh-7 cells, a human-liver cell line commonly used to study infection of SARS viruses ${ }^{34}$, whereas control pseudoparticles without the spike protein of SARS-CoV-2 did not infect (Supplementary Fig. 5b). Vesicular stomatitis virus (VSV) GP protein pseudoparticles (VSVpp) were also generated and used in parallel studies to exclude toxic and non-specific effects of SARS-CoV-2 entry inhibitors ${ }^{20,31}$.

As the SARS-CoV-2pp only permit the analysis of the effects of drugs on cell entry (that is, they do not replicate), we focused these efforts on identifying drugs that might prevent initial SARS-CoV-2 infection and hence could be used for prophylaxis of COVID-19 rather than as therapeutics. A secondary goal was to demonstrate that these types of studies could be carried out under BSL2 conditions as this could help to quickly bring other Organ Chip researchers into this field who similarly do not have access to BSL3 facilities, given the dire challenge at hand.

We first used the Huh-7 cells in a 96-well-plate assay format to test the effects of multiple drugs that have been approved by the
US Food and Drug Administration (FDA) for other medical indications-including chloroquine, hydroxychloroquine, amodiaquine, toremifene, clomiphene, arbidol, verapamil and amiodaroneon SARS-CoV-2pp entry. These drugs were chosen based on the hypothesis that they might have broad-spectrum antiviral activity because they have been shown to inhibit infection by other SARS, influenza and Ebola viruses in previous publications $s^{35-37}$. All of these drugs demonstrated dose-dependent inhibition of SARS-CoV-2pp entry in Huh-7 cells (Fig. 4a) without producing any detectable cell toxicity when added at 1 and $5 \mu \mathrm{M}$ simultaneously with the virus and cultured for $72 \mathrm{~h}$ (Supplementary Fig. 6). These results were promising; however, Huh-7 cells express ACE2 at low levels ${ }^{38}$ and do not express TMPRSS2 (refs. ${ }^{34,39}$ ). In addition, this cell line was derived from a human liver tumour, whereas SARS-CoV-2 preferentially targets lungs in humans.

Thus, to test the clinical translation potential of the drugs that were active in the Huh-7 cell assay, we evaluated their ability to prevent SARS-CoV-2pp infection in the more highly differentiated and physiologically relevant human Airway Chips. SARS-CoV-2pp were introduced into the air channel of the Airway Chips to mimic human infection by airborne SARS-CoV-2. High levels of the viral pol gene encoded by the SARS-CoV-2pp were detected in lung-airway epithelial cells in chips infected by SARS-CoV-2pp within $48 \mathrm{~h}$ but not in control chips that were inoculated with pseudoparticles that lacked the SARS-CoV-2 spike protein (Supplementary Fig. 7a). Infection with SARS-CoV-2pp was also blocked by a neutralizing antibody that targets the receptor-binding domain of SARS-CoV-2 (Supplementary Fig. 7b), confirming that entry of the pseudotyped SARS-CoV-2 virus into the epithelial cells of the human Airway Chip is mediated specifically by the SARS-CoV-2 S protein. The ability of SARS-CoV-2pp to infect human airway epithelial cells on-chip efficiently is consistent with our finding that these highly differentiated lung cells express high levels of its ACE2 receptor as well as TMPRSS2 (Fig. 1d-f and Supplementary Fig. 1d), which mediate the cellular entry of native SARS-CoV-2 virus ${ }^{20,21}$. In addition, immunofluorescence microscopy analysis confirmed that the SARS-CoV2pp preferentially infected ciliated cells in the human Airway Chip (Supplementary Fig. 7c), as native SARS-CoV-2 virus does in vivo ${ }^{21}$.

Next, we pretreated the human Airway Chips by perfusing their vascular channel with amodiaquine, toremifene, clomiphene, chloroquine, hydroxychloroquine, arbidol, verapamil or amiodarone for $24 \mathrm{~h}$ at clinically relevant levels similar to their maximum concentration $\left(C_{\max }\right)$ in blood reported in humans (Table 1) to mimic systemic distribution after oral administration, which is a novel feature of microfluidic Organ Chips ${ }^{16}$. SARS-CoV-2pp were then introduced into the airway channel and incubated statically while continuously flowing the drug through the vascular channel for an additional $48 \mathrm{~h}$. Quantitation of viral mRNA using quantitative PCR with reverse transcription (RT-qPCR) revealed that only three of these drugs-amodiaquine, toremiphene and clomiphene-significantly reduced viral entry (by 59.1, 51.1 and $28.1 \%$, respectively; Fig. 4b) without producing detectable cytotoxicity (Supplementary Fig. 6b) under these more clinically relevant experimental conditions. Importantly, hydroxychloroquine, chloroquine and arbidol, which were active in Huh-7 cells but had no effect on SARS-CoV2 pp entry in our human Airway Chips, also failed to demonstrate clinical benefits in human clinical trials ${ }^{1,2,40}$. When administered to patients, the most potent drug amodiaquine is rapidly transformed (half life $\left(T_{1 / 2}\right)$ of about $5 \mathrm{~h}$ ) into its active metabolite, desethylamodiaquine, which has a much longer $T_{1 / 2}$ (approximately 9-18 days $)^{41}$. When desethylamodiaquine was administered at a clinically relevant dose $(1 \mu \mathrm{M}$; Table 1$)$ in the human Airway Chips, it also reduced entry of the pseudotyped SARS-CoV-2 viral particles by approximately $60 \%$ (Supplementary Fig. 8), which suggests that both amodiaquine and its metabolite are active inhibitors of SARS-CoV-2 S protein-dependent viral entry. 
a
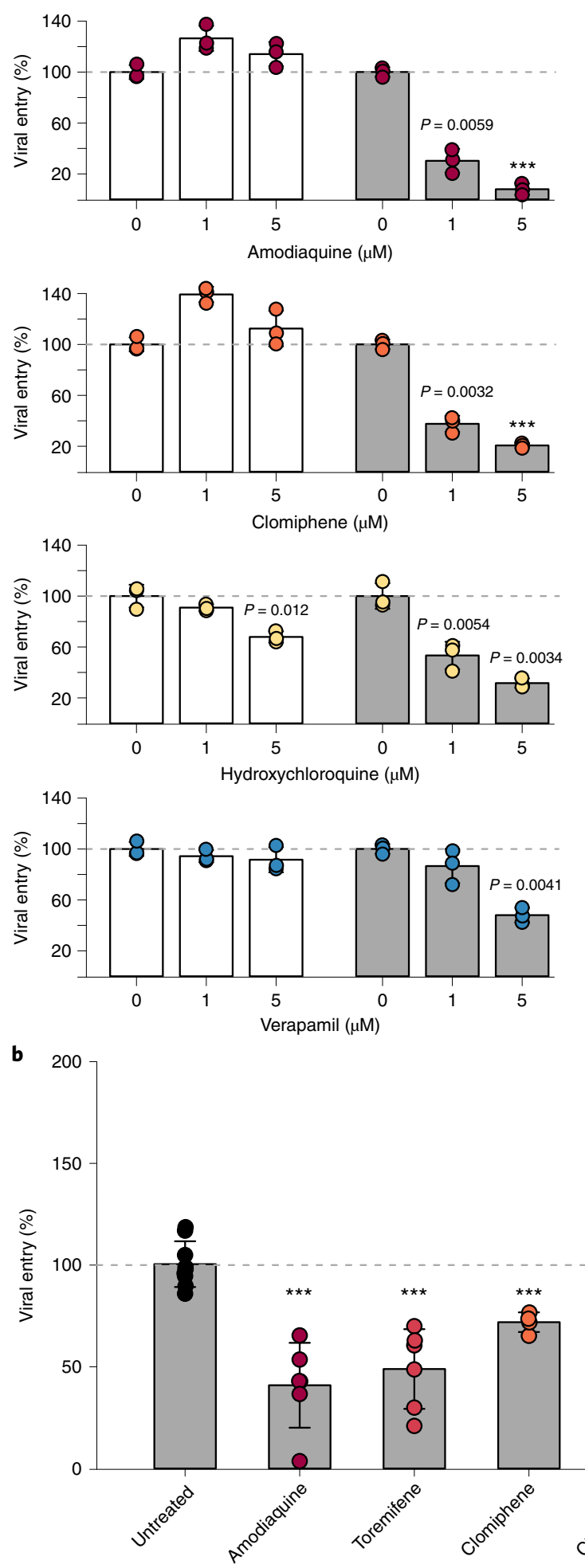

SARS-CoV-2pp
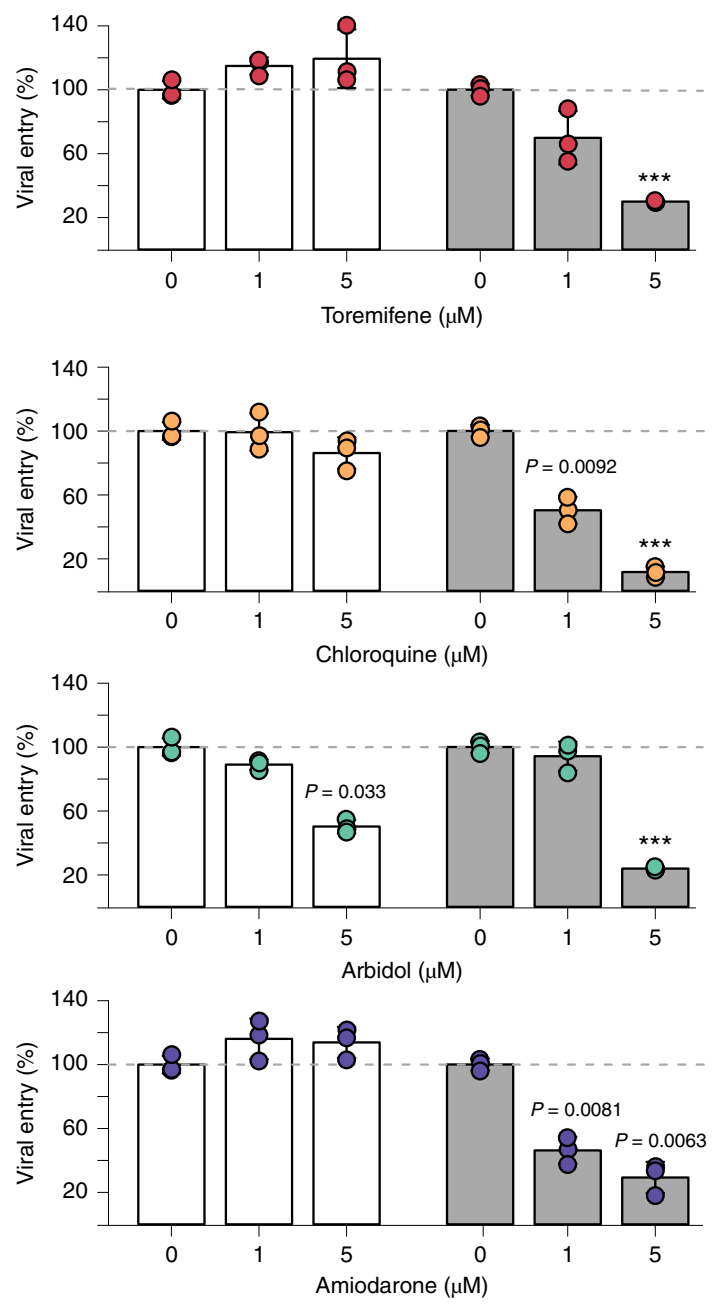

Fig. 4 | Effects of FDA-approved drugs on pseudotyped SARS-CoV-2 virus entry in Huh-7 cells versus human Airway Chips. a, Inhibitory effects of amodiaquine, toremifene, clomiphene, chloroquine, hydroxychloroquine, arbidol, verapamil and amiodarone when added at 0,1 or $5 \mu \mathrm{M}$ to Huh-7 cells infected with SARS-CoV-2pp for $72 \mathrm{~h}$. The number of pseudoparticles in the infected cells was quantified by measuring luciferase activity; viral entry in untreated cells was set as 100\% (dashed horizontal line). VSVpp were tested in parallel to exclude toxic and non-specific effects of the tested drugs. b. Efficacy of the same drugs in human Airway Chips infected with SARS-CoV-2pp. The drugs were delivered into the apical and basal channels of the chip at their respective $C_{\max }$ in human blood, and the chips were infected with SARS-CoV-2pp one day later and maintained in the continued presence of the drugs for two more days. The epithelium from the chips was collected for the detection of the viral pol gene by RT-qPCR; viral entry in untreated chips was set as 100\% (dashed horizontal line). Data represent the mean \pm s.d.; $n=3$ (a) and 3-6 (b) biological replicates; ${ }^{\star \star \star} P<0.001$. 


\begin{tabular}{|c|c|c|}
\hline Drug & $C_{\max }\left(\mathrm{ng} \mathrm{ml}^{-1}\right)$ & $C_{\max }(\mu \mathrm{M})$ \\
\hline Amodiaquine & 575 & 1.24 \\
\hline Toremifene & 1,211 & 2.98 \\
\hline Clomiphene & 500 & 0.83 \\
\hline Chloroquine & 960.5 & 1.91 \\
\hline Hydroxychloroquine & 422 & 1.25 \\
\hline Arbidol & 2,160 & 3.89 \\
\hline Verapamil & $287 \pm 105$ & 0.81 \\
\hline Amiodarone & $13,660 \pm 3,410$ & 20.04 \\
\hline Desethylamodiaquine & $329-828$ & 1.00 \\
\hline
\end{tabular}

Given that amodiaquine was an effective inhibitor of viral entry of SARS-CoV-2, whereas the related antimalarial drugs hydroxychloroquine and chloroquine were not, we performed quantitative mass spectrometry to compare the effects of these three drugs on the proteome of airway epithelial cells. Proteomics analysis revealed that amodiaquine triggered distinct and broader perturbations in the host proteome compared with the other related antimalarial drugs (Fig. 5a), with the most differentially affected proteins being related to the regulation of cilia (Fig. 5b) and expression of lysosomal proteins (Fig. 5c), which may be responsible for it having greater effects against viral entry.

Amodiaquine and desethylamodiaquine inhibit SARS-CoV-2 infection in vitro and in vivo. Finally, we tested the ability of amodiaquine, the most potent prophylactic drug identified in the Airway Chip, and its metabolite desethylamodiaquine to inhibit infection by native GFP-expressing SARS-CoV-2 virus at a multiplicity of infection (MOI) of 0.1 in Vero E6 cells and found that both compounds inhibited infection in a dose-dependent manner (Supplementary Fig. 9), with half-maximal inhibitory concentrations $\left(\mathrm{IC}_{50}\right)$ of $7.5+4.5 \mu \mathrm{M}$ for amodiaquine and $9.9+4.1 \mu \mathrm{M}$ for its active metabolite. This finding is consistent with our own earlier findings using SARS-CoV-2 without GFP and similar results in Vero E6 cells from other laboratories ${ }^{42,43}$. In addition, amodiaquine reduced the viral load by about three orders of magnitude in ACE2-expressing human A549 lung epithelial cells infected with native SARS-CoV-2 when administered at $10 \mu \mathrm{M}$ (Fig. 6a).

Given this potent inhibitory activity against native SARS-CoV-2, we then evaluated amodiaquine in a hamster COVID-19 prevention model in which the drug was first administered subcutaneously $\left(50 \mathrm{mg} \mathrm{kg}^{-1}\right)$ one day before the animals were infected intranasally with SARS-CoV-2 virus $\left(1 \times 10^{3}\right.$ plaque-forming units (p.f.u.) $)$ and then treated daily with the same dose for three additional days. The dosing regimen was selected based on a PK study for amodiaquine that was carried out in healthy hamsters. A single dose of amodiaquine injected subcutaneously at this dose produced a $C_{\max }$ of approximately 3.2 and $0.7 \mu \mathrm{M}$ for amodiaquine and its active metabolite desethylamodiaquine, respectively; the $T_{1 / 2}$ for amodiaquine was $18.1 \mathrm{~h}$ and that of its active metabolite was significantly longer than the time period of analysis (that is, one day; Supplementary Fig. 10a,b). Analysis of the drug concentrations $24 \mathrm{~h}$ after dosing revealed considerable exposures of amodiaquine and desethylamodiaquine in the lung, kidney and intestine (Supplementary Fig. 10c), with levels in tissues relative to plasma enhanced 21-138-fold for amodiaquine and 8-45-fold for desethylamodiaquine. These PK results, including the extended $T_{1 / 2}$ and tissue concentration for both the parent compound and metabolite, are consistent with the results of previous PK studies in humans ${ }^{41}$.
Importantly, prophylaxis of infected hamsters with amodiaquine beginning one day before infection with daily treatment over the following three days resulted in a reduction of approximately $70 \%$ in the SARS-CoV-2 viral load (measured by RT-qPCR of the viral nucleocapsid (N) transcript) when measured on the third day after the viral challenge (Fig. 6b), which is when these animals exhibit fulminant infection and high viral loads, as previously demonstrated in the hamster model ${ }^{44-46}$. Immunohistochemical analysis of the lungs of these animals confirmed that treatment with amodiaquine resulted in a significant reduction in the expression of the SARS-CoV-2 N protein in these tissues (Fig. 6c). To explore the potential use of amodiaquine in the prevention of the spread of COVID-19 within populations, we then carried out studies using a SARS-CoV-2 animal-to-animal transmission model, in which vehicle or healthy animals treated with amodiaquine for one day were placed in the same cage with animals that had been infected with the SARS-CoV-2 virus $\left(1 \times 10^{3}\right.$ p.f.u. $)$ one day earlier. In vehicle controls, this experimental set-up resulted in $100 \%$ transmission of infection within two days of exposure to the infected animals. In contrast, the same amodiaquine treatment regimen resulted in a $90 \%$ reduction in the viral load, as measured by quantifying the $\mathrm{N}$ transcript in lungs after exposing healthy animals to SARS-CoV-2-infected hamsters (Fig. 6d, left). These results were further corroborated in an independent experiment where amodiaquine-treated animals showed a decrease in the viral titres of more than one log, measured by plaque assays, when compared with the vehicle (Fig. 6d, right). As amodiaquine is commonly clinically administered as an oral dose, we repeated these studies with drug administered through oral gavage at a dose $\left(75 \mathrm{mg} \mathrm{kg}^{-1}\right)$ that produced similar PK parameters to those observed with the subcutaneous administration $\left(C_{\max }\right.$ for amodiaquine and desethylamodiaquine of approximately 1.8 and $4.5 \mu \mathrm{M}$, respectively; the $T_{1 / 2}$ for amodiaquine was $12.8 \mathrm{~h}$ and again greater than $24 \mathrm{~h}$ for its active metabolite; Supplementary Fig. 11a,b). However, oral administration resulted in even higher levels of the drugs in tissues compared with plasma (enhanced 29-331-fold for amodiaquine and 8-119-fold for desethylamodiaquine; Supplementary Fig. 11c). Importantly, pretreatment with oral amodiaquine for one day prevented infection of native SARS-CoV-2 (Fig. 6e) to a similar degree to subcutaneous administration (Fig. 6b). In addition, when we used the same prevention model to test hydroxychloroquine at a dose previously shown to produce a clinically relevant level of lung exposure in hamsters $\left(50 \mathrm{mg} \mathrm{kg}^{-1}\right)^{41}$, it did not exhibit any significant inhibitory activity relative to the control or amodiaquine-treated animals (Fig. 6e), similar to what we observed in our human Organ Chip model (Fig. 4b). Furthermore, RNA-sequencing analysis of infected hamsters treated with oral amodiaquine revealed that the drug treatment resulted in a significant downregulation of genes associated with the inflammatory response, including those involved in signalling through TNF-a and NF- $\mathrm{BB}$, IL-6-JAK-STAT3 and interferon- $\mathrm{X}$ (Supplementary Fig. 12).

Finally, given the robustness of these responses, we tested the ability of amodiaquine to inhibit SARS-CoV-2 infection when administered in a treatment mode, beginning one day after infection. These studies revealed that post-infection treatment with amodiaquine produces similar inhibition of infection by about $70 \%$ at day 3, with complete clearance of detectable viral $\mathrm{N}$ transcripts in the lung by day 7 (Fig. 6f). These results confirm that the effects we observed were not simply due to a time delay in the response. Together, these results confirm that the antiviral activities exhibited by amodiaquine in the human Airway Chips translate to the in vivo setting and suggest that oral amodiaquine may provide substantial protection when administered prophylactically or therapeutically.

\section{Discussion}

Together, our data show that human Organ Chips, such as the Airway Chip, can be used to rapidly identify existing approved 
a

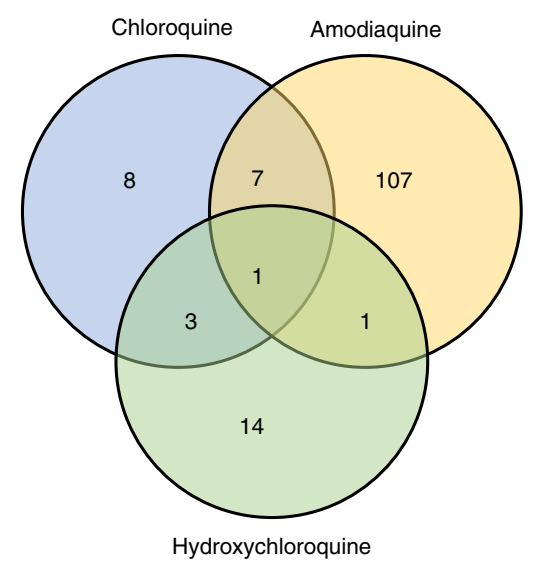

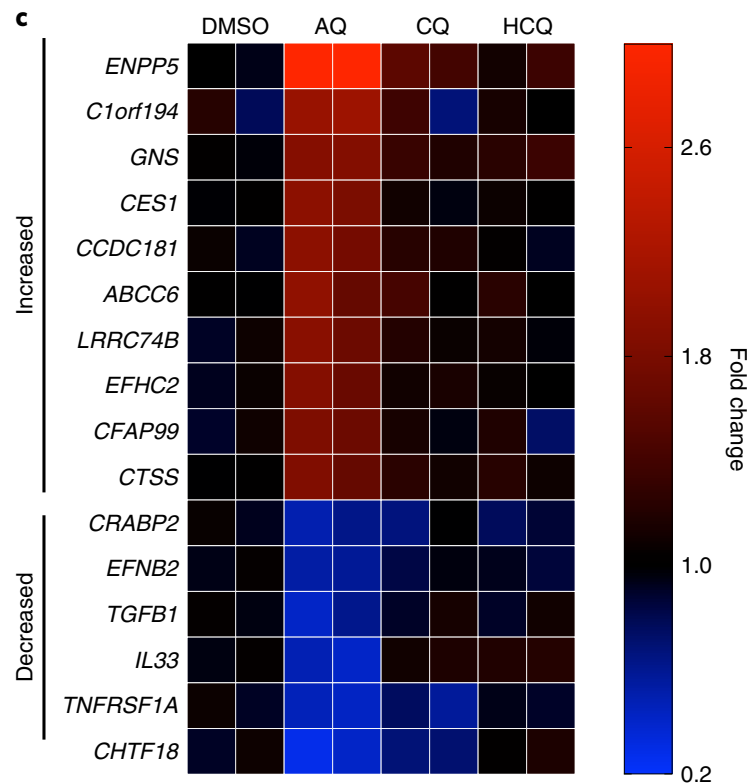

b

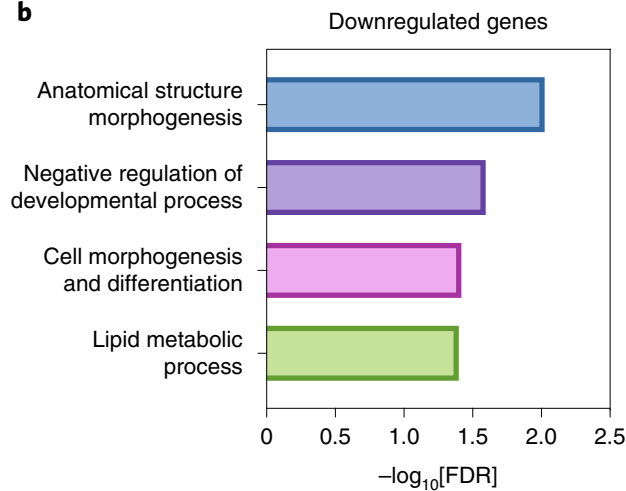

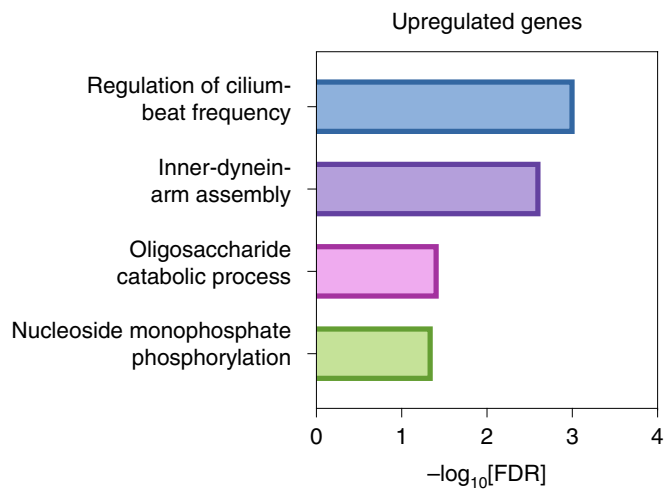

Fig. 5 | Divergent effects on the host proteome induced by amodiaquine versus hydroxychloroquine and chloroquine. a, Venn diagram showing the number of common and unique protein perturbations by the three drugs. b. Gene Ontology analysis for proteins downregulated (left) and upregulated (right) by amodiaquine. FDR, false-discovery rate. c, Heatmap of the top differentially affected proteins (shown by gene name). DMSO, dimethyl sulfoxide; $\mathrm{AQ}$, amodiaquine; $\mathrm{CQ}$, chloroquine; $\mathrm{HCQ}$, hydroxychloroquine.

drugs that may be repurposed for pandemic-virus applications in crisis situations that require the accelerated development of potential therapeutic and prophylactic interventions. Our work on repurposing of therapeutics for COVID-19 was initiated on 13 January 2020 (one day after the sequence of the viral genome was published in GenBank ${ }^{32}$ ). Our first results with drugs in Airway Chips were obtained three weeks later and we published a preprint describing our findings on 13 April 2020 (ref. ${ }^{47}$ ). Subsequent preprints and publications from other laboratories also have described the utility of using Organ Chips to study infection and inflammation responses caused by SARS-CoV-2 infection ${ }^{48-50}$. However, all of these reports have used chips lined with human primary alveolar epithelial cells or cell lines rather than airway epithelium, which is the primary target of initial infection in vivo, and none explored drug repurposing. In contrast to these studies, we also applied drugs using dynamic fluid flow in our Airway Chips, which enables the human lung cells to be treated in vitro with more clinically relevant drug exposures similar to those obtained in human patients. Although animal models remain the benchmark for validation of therapeutics to move to humans, it is also important to note that human Organ Chips are now being explored as viable alternatives to animal models ${ }^{16}$ and regulatory agencies are encouraging pharmaceutical and biotechnology companies to make use of data from Organ Chips and other microphysiological systems in their regulatory submissions ${ }^{51}$.

The human Airway Chip used here differed from a previously published version of the device ${ }^{17}$ in that it contains a membrane with different chemistry (poly-dimethylsiloxane versus poly-ethylene terephthalate), material properties (flexible versus rigid) and larger pores $(7 \mathrm{~mm}$ versus $0.4 \mathrm{~mm}$ ). However, validation studies involving infection with influenza A virus confirmed that similarly high levels of airway epithelial cell differentiation and organ-level pathophysiology can be recapitulated on-chip. Moreover, the level of clinical mimicry exhibited by these chips far surpasses that demonstrated by any other type of in vitro model of the lung airway. For example, we were able to replicate the effects of infection of influenza A H1N1 virus on pulmonary vascular leakage, increased virulence and neutrophil recruitment for $\mathrm{H} 3 \mathrm{~N} 2$ versus $\mathrm{H} 1 \mathrm{~N} 1$, enhanced chemokine and cytokine levels for H5N1 compared with H1N1 and H3N2 (despite lower viral loads) and a protective role of neutrophils (provided by clearance of virus) - all of which have been observed in vivo to contribute to disease pathogenesis. We also observed that neutrophil infiltration following influenza infection further increased cytokine production and caused more barrier damage in the Airway Chips. However, it is worth noting that neutrophils 
a

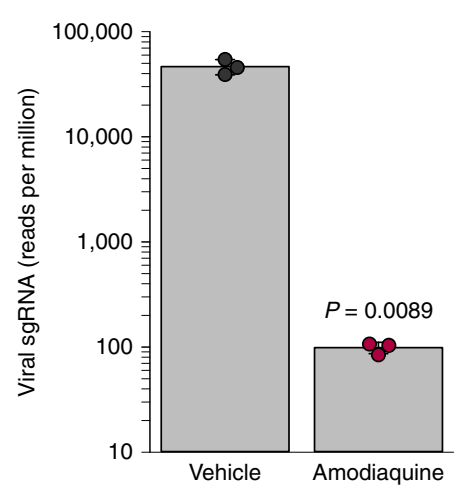

d

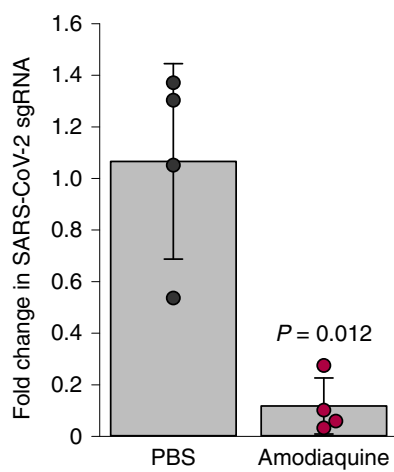

b
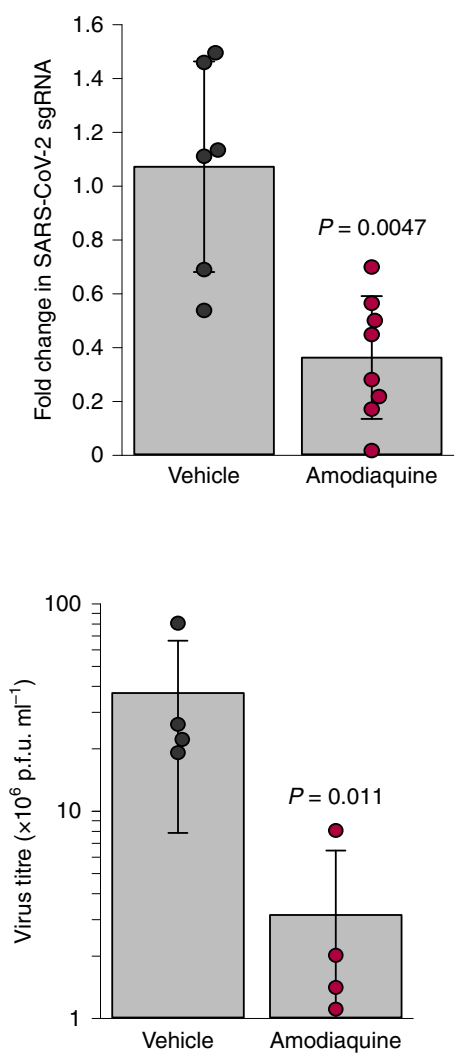

c

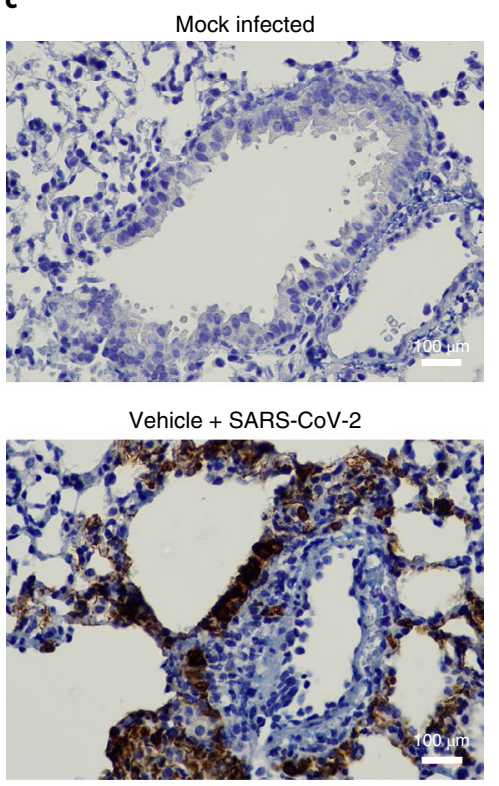

Amodiaquine + SARS-CoV-2

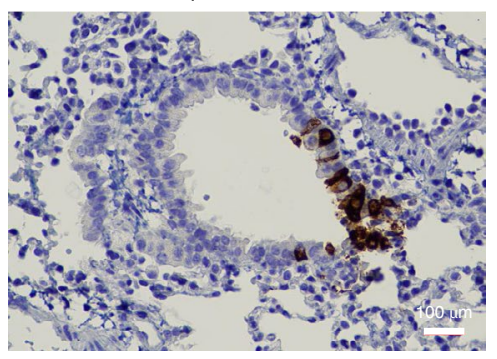

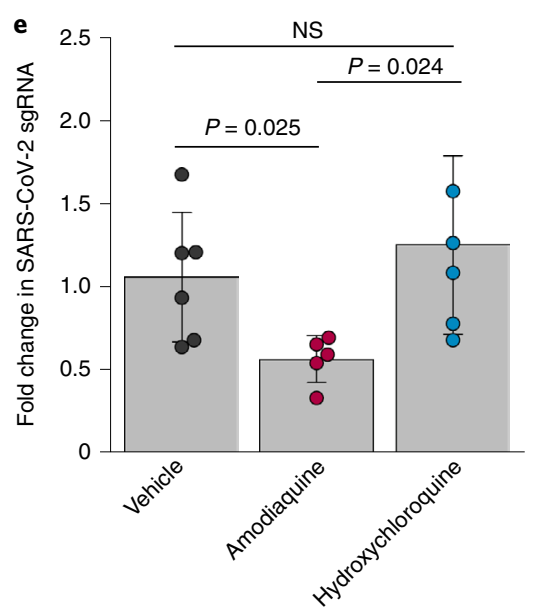
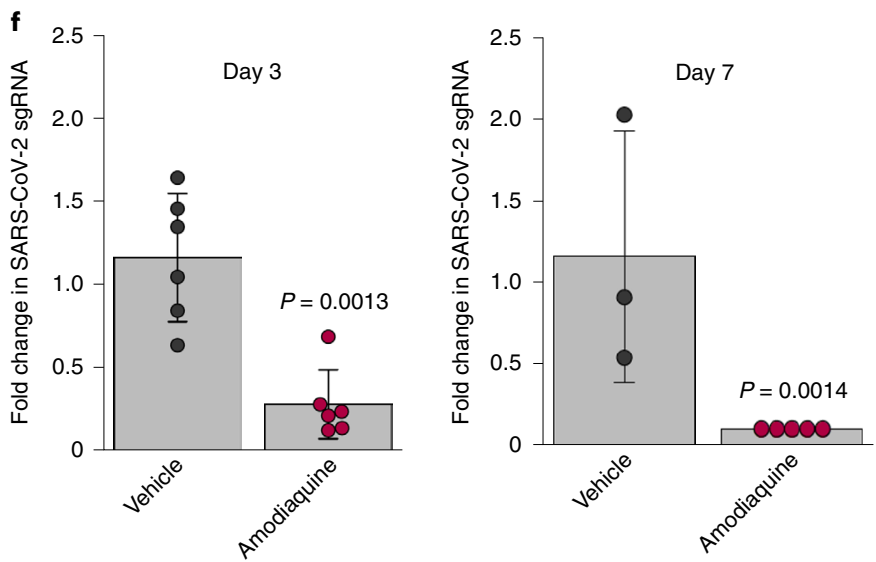

Fig. 6 | Inhibition of infection by native SARS-CoV-2 virus in vitro and in vivo. a, In vitro inhibition of wild-type SARS-CoV-2 infection in ACE2-expressing A549 cells by $10 \mu \mathrm{M}$ amodiaquine. b, Prevention of SARS-CoV-2 infection in vivo. Reduction of the viral load in the lungs of hamsters treated once a day with amodiaquine $\left(50 \mathrm{mg} \mathrm{kg}^{-1}\right)$ administered subcutaneously beginning one day before the intranasal administration of SARS-CoV-2 virus ( $1 \times 10^{3}$ p.f.u.), as measured by RT-qPCR for subgenomic RNA encoding the SARS-CoV-2 N protein. c, Haematoxylin (blue)- and SARS-CoV-2 N (brown)-stained histological sections of the lungs of animals that were mock treated, infected with SARS-CoV-2 and treated with vehicle alone or infected with SARS-CoV-2 and treated with amodiaquine ( $50 \mathrm{mg} \mathrm{kg}^{-1}$ subcutaneously). Scale bars, $100 \mu \mathrm{m}$. d, Inhibition of SARS-CoV-2 transmission in vivo. Reduction of the viral load in the lungs of hamsters treated with subcutaneous amodiaquine ( $50 \mathrm{mg} \mathrm{kg}^{-1}$ ) once a day for four days beginning one day before co-caging with SARS-CoV-2-infected animals. Levels of RNA encoding the SARS-CoV-2 N protein determined by RT-qPCR (left) and p.f.u. concentrations (right) in the lung homogenate. e, Prevention of SARS-CoV-2 infection in vivo. Reduction of the viral load in the lungs of hamsters treated once a day with oral amodiaquine $\left(75 \mathrm{mg} \mathrm{kg}^{-1}\right)$ versus oral hydroxychloroquine $\left(50 \mathrm{mg} \mathrm{kg}^{-1}\right)$ beginning one day before the intranasal administration of SARS-CoV-2 virus $\left(1 \times 10^{3}\right.$ p.f.u.), as measured by RT-qPCR for subgenomic RNA encoding the SARS-CoV-2 N protein. NS, not significant. f, Treatment of SARS-CoV-2 infection in vivo. Reduction of the viral load, measured on days 3 (left) and 7 (right), in the lungs of hamsters treated daily with oral amodiaquine $\left(75 \mathrm{mg} \mathrm{kg}^{-1}\right)$ beginning one day after the intranasal administration of SARS-CoV-2 virus $\left(1 \times 10^{3}\right.$ p.f.u. $)$, as measured by RT-qPCR for subgenomic RNA encoding the SARS-CoV-2 N protein. Data represent the mean \pm s.d.; $n=3(\mathbf{a}), 8(\mathbf{b}), 4(\mathbf{d}), 6(\mathbf{e})$ and 3-6 (f) biological replicates. 
can either protect or damage host barriers depending on the viral strain, infection titres, pre-existing conditions and host genetics ${ }^{52,53}$, and these are precisely the types of questions that can be addressed using Organ Chips in the future.

Importantly, studies using these human Airway Chips led to the identification of multiple approved drugs that could serve as prophylactics and therapeutics against viral pandemics. Again, we first demonstrated clinical mimicry by showing that oseltamivir displayed efficacy and a therapeutic time window against influenza A infection on-chip that were similar to those observed in human patients. In addition, we discovered that the anticoagulant drug nafamostat significantly extended the treatment-time window of oseltamivir from two to four days after infection by influenza virus, which could have great clinical relevance given that most patients do not begin treatment until days after they are infected. This human Organ Chip model also predicted the inability of chloroquine, hydroxychloroquine and arbidol to work in animals and human patients ${ }^{1,2,54}$, in contrast with what was reported in cell lines $^{42,55,56}$. Although the drugs were administered at levels similar to their $C_{\max }$ here to compare the relative potencies, one caveat is that we did not quantify drug absorption or protein binding in this study. Importantly, by carrying out mass-spectrometry measurements of the drug levels in these devices, full PK profiles can be recapitulated in these Organ Chip models ${ }^{8}$, which should further aid clinical translation in the future.

The relevance for the COVID-19 crisis was also demonstrated by using the human Airway Chip to identify amodiaquine as a putative therapy for SARS-CoV-2 that works both in vitro and in vivo. Amodiaquine is an antimalarial drug related to chloroquine and hydroxychloroquine ${ }^{57}$. This drug was the most potent inhibitor of SARS-CoV-2pp entry into human airway cells, producing approximately $60 \%$ inhibition when administered under flow at $1.24 \mu \mathrm{M}$, which should be clinically achievable in the plasma of patients with malaria who receive a $300-\mathrm{mg}^{\text {administration }}{ }^{58}$ as well as in tissues such as lung where the drug and its metabolite concentrate ${ }^{37}$. Importantly, further investigation of amodiaquine revealed that both this drug and its active metabolite (desethylamodiaquine) do indeed inhibit native SARS-CoV-2 infection in vitro and in vivo when administered either subcutaneously or orally, as it is commonly administered in patients. Moreover, we found that similar inhibition of SARS-CoV-2 infection could be obtained by administering amodiaquine in either prevention or treatment modes. Thus, these findings suggest that the microfluidic human Organ Chip model, combined with existing preclinical cell-based and animal assays, offers a potentially more clinically relevant test bed for the accelerated discovery of anti-COVID-19 drugs. However, to most effectively focus on the discovery of COVID-19 therapeutics rather than prophylactics, it will be necessary to integrate human Organ Chips into BSL3 laboratories where experiments with native SARS-CoV-2 can be carried out in the future.

When considering the repurposing of approved drugs for COVID-19, it is important to recognize that every drug has its own distinct therapeutic and toxicity profile that must be taken into consideration. Amodiaquine has been widely and safely used for prophylaxis and treatment of malaria for over 60 years. It is currently used in low-resource nations where the World Health Organization recommends it be used in combination with artesunate for the chemoprophylaxis of malaria and as a second-line acute treatment for uncomplicated Plasmodium falciparum-resistant malaria. Interestingly, the amodiaquine-artesunate drug combination has also been reported to lower the risk of death from Ebola virus disease ${ }^{59}$. Amodiaquine has been given a black-box warning by the FDA in the United States due to the rare occurrence of agranulocytosis and liver damage with high doses or prolonged treatment ${ }^{60}$; however, it continues to be approved for use in other nations and remains well tolerated among African populations, where it is commonly administered for a short duration (three-day course). The short course is possible because the $T_{1 / 2}$ of desethylamodiaquine, the active metabolite of amodiaquine, is very long (in the order of 9-18 days) and it concentrates in organs, including the lung ${ }^{41}$.

Given the alarming rate at which the SARS-CoV-2 pandemic is spreading, clinicians must seriously consider the relative risks and benefits of using an existing approved drug as a new COVID-19 therapy with specific patient populations (for example, male versus female, young versus old, White versus Black, and so on) before initiating any trial in their local communities. Our findings raise the possibility that amodiaquine could be explored as a chemoprophylaxis therapy to prevent the spread of COVID-19. This type of prevention therapy could help people return to work by treating healthy patients for three days, which could then offer protection for approximately two weeks. In addition, it could be administered orally after patients learn that they are infected at home or once they enter the hospital, either alone or in combination with other COVID-19 therapies. If amodiaquine were to be used for COVID19 , it would be critical to select the patient populations carefully and carry out the appropriate clinical assessments (for example, blood and liver-function tests) before and during administration of the drug. Amodiaquine therapy for COVID-19 may be particularly valuable in low-resource nations, where this inexpensive drug is readily available and more expensive alternative therapies, and even vaccines, are not easily accessible. In fact, supported in part by the findings presented in our earlier preprint ${ }^{47}$, human Phase 2 clinical trials for treatment of COVID-19 using amodiaquine were recently initiated by Medicines for Malaria Venture and Wits University, and by Drugs For Neglected Drugs initiative through their ANTICOV trials, in Africa.

The current COVID-19 pandemic and potential future ones caused by influenza viruses or other coronaviruses represent imminent dangers and major ongoing public health concerns. When it comes to repurposing existing antiviral agents, every experimental assay has its limitations. However, our results suggest that combining multiplexed cell-based assays with lower throughput or higher content, human Organ Chips that recapitulate clinically relevant human organ-level responses as well as animal models, and focusing on compounds that are active in all models, could provide a fast track to identify potential treatments for the current COVID-19 crisis that have a higher likelihood of working in human patients. This discovery pipeline may be equally valuable to combat unforeseen biothreats, such as new pandemic influenza or coronavirus strains.

\section{Methods}

Human Airway Chip culture. Microfluidic two-channel Organ Chip devices containing membranes with $7-\mu \mathrm{m}$ pores were obtained from Emulate Inc. Each microdevice contains two adjacent parallel microchannels (apical, $1 \mathrm{~mm}$ wide $\times 1 \mathrm{~mm}$ high; basal, $1 \mathrm{~mm}$ wide $\times 0.2 \mathrm{~mm}$ high; length of overlapping channels, $16.7 \mathrm{~mm}$ ) separated by the porous membrane. Similar results were obtained in some studies not involving immune-cell recruitment using two-channel devices fabricated from poly-dimethylsiloxane with a PET membrane containing $0.4-\mu \mathrm{m}$ pores, as used in past Airway Chip studies ${ }^{17}$. Before cell plating, both channels of these devices were washed with $70 \%$ ethanol, filled with $0.5 \mathrm{mg} \mathrm{ml}^{-1}$ ER1 solution in ER2 buffer (Emulate Inc.) and placed under an ultraviolet lamp (Nailstar, NS01 -US) for $20 \mathrm{~min}$ to activate the surface for protein coating. The channels were then washed sequentially with ER2 buffer and PBS. The porous membranes were coated on both sides with collagen type IV from human placenta $\left(0.5 \mathrm{mg} \mathrm{m}^{-1}\right.$ in water; Sigma-Aldrich) at room temperature overnight. The solution was aspirated from the chip, which was then used for seeding cells.

Primary human lung bronchial-airway epithelial basal stem cells (Lonza, cat. no. CC-2540S; obtained from healthy donors 448571, 446317, 623950, 485960 and 672447 ) were expanded in $75-\mathrm{cm}^{2}$ tissue-culture flasks using airway epithelial cell growth medium (Promocell) to $60-70 \%$ confluency. Primary human pulmonary microvascular endothelial cells (Cell Biologics) were expanded in $75-\mathrm{cm}^{2}$ tissue-culture flasks using human endothelial cell growth medium (Cell Biologics) to $70-80 \%$ confluency.

To create the human Airway Chips, endothelial cells $\left(2 \times 10^{7}\right.$ cells ml $\left.{ }^{-1}\right)$ were first seeded in the bottom channel by inverting the chip for $4 \mathrm{~h}$ in human endothelial cell growth medium, followed by inverting the chip again and seeding 
of the top channel with the lung bronchial-airway epithelial basal stem cells $\left(2.5 \times 10^{6} \mathrm{cells} \mathrm{ml}^{-1}\right)$ for $4 \mathrm{~h}$ in airway epithelial cell growth medium. The respective medium for each channel was refreshed and the chips were incubated overnight under static conditions at $37^{\circ} \mathrm{C}$ with $5 \% \mathrm{CO}_{2}$. The adherent cells were then continuously perfused with the respective cell-culture medium using an IPC-N series peristaltic pump (Ismatec) or Zoe (Emulate) at a volumetric flow rate of $60 \mu \mathrm{h}^{-1}$. After 5-7 days, the apical medium was removed while allowing air to fill the channel to establish an ALI and the airway epithelial cells were cultured for 3-4 additional weeks while being fed only by constant flow of PneumaCult-ALI medium (StemCell) supplemented with 0.1\% VEGF, 0.01\% EGF and $1 \mathrm{mM} \mathrm{CaCl}$ from an Endothelial cell medium kit (Cell Biological, M1168) through the bottom vascular channel. The chips were cultured in an incubator containing $5 \% \mathrm{CO}_{2}$ and $16-18 \% \mathrm{O}_{2}$ at $85-95 \%$ humidity, and the apical surface of the epithelium was rinsed with PBS once a week to remove the cellular debris and mucus. Highly differentiated human airway structures and functions can be maintained in the human Airway Chip for more than two months.

\section{Immunofluorescence microscopy. Cells were washed with PBS through the} apical and basal channels, fixed with $4 \%$ paraformaldehyde (Alfa Aesar) for 20-25 min and then washed with PBS before being stored at $4{ }^{\circ} \mathrm{C}$. Fixed tissues were permeabilized on-chip with $0.1 \%$ Triton X-100 (Sigma-Aldrich) in PBS for $5 \mathrm{~min}$, exposed to PBS with $10 \%$ goat serum (Life Technologies) and $0.1 \%$ Triton X-100 for $30 \mathrm{~min}$ at room temperature, and then incubated with primary antibodies (Supplementary Table 2) diluted in incubation buffer (PBS with 1\% goat serum and $0.1 \%$ Triton $\mathrm{X}-100$ ) overnight at $4{ }^{\circ} \mathrm{C}$, followed by incubation with the corresponding secondary antibodies (Supplementary Table 2) for $1 \mathrm{~h}$ at room temperature; the nuclei were counterstained with DAPI (Invitrogen) after the secondary-antibody staining. Fluorescence imaging was carried out using a confocal laser-scanning microscope (Leica SP5 X MP DMI-6000) and image processing was done using the Imaris software (Bitplane).

Barrier-function assessment. To measure the tissue barrier permeability, $50 \mu \mathrm{l}$ cell medium containing Cascade blue $\left(607 \mathrm{Da} ; 50 \mu \mathrm{g} \mathrm{ml}^{-1}\right.$; Invitrogen) was added to the bottom channel and $50 \mu \mathrm{l}$ cell medium was added to the top channel. The fluorescence intensity of the medium of the top and bottom channels was measured $2 \mathrm{~h}$ later in three different human Airway Chips. The apparent permeability was calculated using the formula: $P_{\text {app }}=J /(A \times \Delta C)$, where $P_{\text {app }}$ is the apparent permeability, $J$ is the molecular flux, $A$ is the total area of diffusion and $\Delta C$ is the average gradient.

Mucus quantification. Mucus present in the airway channel was isolated by infusing $50 \mu \mathrm{l}$ PBS into the upper channel of the Airway Chip, incubating for $1 \mathrm{~h}$ at $37^{\circ} \mathrm{C}$, and then collecting the fluid and storing it at $-80^{\circ} \mathrm{C}$ before analysis, as previously described ${ }^{6}$. Quantification of mucus production was carried out by quantifying the Alcian blue staining (Thermo Fisher Scientific) and comparison to serially diluted standards of mucin (Sigma-Aldrich) in PBS.

RT-qPCR. Total RNA was extracted from differentiated human Airway Chips, pre-differentiated lung bronchial-airway epithelial cells or MDCK cells using TRIzol (Invitrogen). Complimentary DNA was then synthesised using an AMV reverse transcriptase kit (Promega) with oligo-dT primer. To determine the cellular gene-expression levels, RT-qPCR was carried out according to the GoTaq qPCR master mix (Promega) with $20 \mu$ of a reaction mixture containing gene-specific primers (Supplementary Table 3 ). The expression levels of the target genes were normalized to GAPDH.

Influenza viruses. The influenza virus strains used in this study are: A/ PR/8/34 (H1N1), GFP-expressing A/PR/8/34 (H1N1), A/WSN/33 (H1N1), A/ Netherlands/602/2009 (H1N1), A/Hong Kong/8/68/ (H3N2), A/Panama/2007/99 (H3N2) and A/Hong Kong/156/1997 (H5N1). A/PR/8/34 (H1N1), GFP-expressing $\mathrm{A} / \mathrm{PR} / 8 / 34(\mathrm{H} 1 \mathrm{~N} 1)$ and $\mathrm{A} / \mathrm{WSN} / 33(\mathrm{H} 1 \mathrm{~N} 1)$ were generated using reverse genetics techniques. The other viruses were obtained from the Centers for Disease Control and Prevention (CDC) or from P. Palese, R. A. M. Fouchier and A. Carcia-Sastre.

Infection of human Airway Chips with influenza virus. Human Airway Chips were infected with influenza viruses by flowing $30 \mu \mathrm{PBS}$ containing the indicated MOI of viral particles into the apical channel, incubating for $2 \mathrm{~h}$ at $37^{\circ} \mathrm{C}$ under static conditions and then removing the medium to re-establish an ALI. To measure virus propagation, the apical channel was incubated with $50 \mu \mathrm{PBS}$ for $1 \mathrm{~h}$ at $37^{\circ} \mathrm{C}$ at various times, and the apical fluid and vascular effluent were then collected from the apical and basal channels, respectively. The fluid collected from the apical channel effluent was subjected to viral-load quantification using the plaque-formation assay, whereas the released cytokines and chemokines were analysed in the effluents from the lower vascular channel. The tissues cultured on-chip were also fixed and subjected to immunofluorescence microscopy analysis.

To test the efficacy of oseltamivir acid, Airway Chips infected with influenza virus $(\mathrm{MOI}=0.1)$ were treated with $1 \mu \mathrm{M}$ oseltamivir acid (Sigma-Aldrich) under flow $\left(60 \mu \mathrm{h}^{-1}\right)$ through the vascular channel. To explore the effects of serine protease inhibitors on influenza infection, nafamostat (Abcam) or Trasylol (G-Biosciences) were delivered into the airway channel of influenza-infected chip $(\mathrm{MOI}=0.1)$. The virus samples were collected two days later for viral-load determination and the vascular effluents were collected for the analysis of cytokines and chemokines. In the treatment-time-window determination experiment, oseltamivir acid $(1 \mu \mathrm{M})$, nafamostat $(10 \mu \mathrm{M})$ or both were added to the influenza H1N1-infected Airway Chips $(\mathrm{MOI}=0.1)$ at the indicated times. Oseltamivir was perfused through the vascular channel, whereas nafamostat was introduced in $20 \mu \mathrm{l}$ PBS and incubated in the airway channel for $48 \mathrm{~h}$. Fluid samples were then collected from both channels to determine the viral load.

Analysis of neutrophil infiltration. Neutrophils isolated from fresh human blood using a Ficoll-Paque PLUS (GE Healthcare) gradient were resuspended in medium at a concentration of $5 \times 10^{6} \mathrm{cells} \mathrm{ml}^{-1}$, which is within the normal range $\left(2.5-7.5 \times 10^{6} \mathrm{cells} \mathrm{ml}^{-1}\right)$ of neutrophils found in human blood. The isolated neutrophils were labelled with CellTracker Red CMTPX (Invitrogen) and injected into the vascular channel of inverted Airway Chips infected with influenza virus $(\mathrm{MOI}=0.1)$ at a flow rate of $50-100 \mu \mathrm{h}^{-1}$ using a syringe pump; $2 \mathrm{~h}$ later, the unbound neutrophils were washed away by flowing cell-free medium through the channel for $24 \mathrm{~h}$. Virus samples were collected by incubating the airway channel with $50 \mu \mathrm{PBS}$ for $1 \mathrm{~h}$ at $37^{\circ} \mathrm{C}$, collecting the fluid and determining the virus load using the plaque assay. The cell layers were fixed on-chip and subjected to immunofluorescence microscopy analysis for the influenza-virus nucleoprotein (Invitrogen) and neutrophils (CD45, BioLegend). Micrographs of four or five random areas of the chips were taken for subsequent quantification of the infiltrated neutrophils. To study the interaction between influenza virus and neutrophils, Airway Chips were infected with GFP-expressing PR8 virus $(\mathrm{MOI}=0.1)$ for 24h. CellTracker Red CMTPX-labelled neutrophils $\left(5 \times 10^{6} \mathrm{cells} \mathrm{ml}^{-1}\right)$ were perfused in medium through the vascular channel of the infected Airway Chips. Immunofluorescence microscopy analysis was carried out at the indicated times.

Plaque-formation assay. The virus titres were determined using plaque-formation assays. Confluent MDCK cell monolayers in 12-well plates were washed with PBS, inoculated with $1 \mathrm{ml}$ of tenfold serial dilutions of influenza virus samples and incubated for $1 \mathrm{~h}$ at $37^{\circ} \mathrm{C}$. After the unabsorbed virus was removed, the cell monolayers were overlaid with $1 \mathrm{ml}$ DMEM medium (Gibco) supplemented with $1.5 \%$ low-melting-point agarose (Sigma-Aldrich) and $2 \mu \mathrm{g} \mathrm{ml}^{-1}$ TPCK-treated trypsin (Sigma-Aldrich). After incubation for $2-4$ days at $37^{\circ} \mathrm{C}$ with $5 \% \mathrm{CO}_{2}$, the cells were fixed with $4 \%$ paraformaldehyde and stained with crystal violet (Sigma-Aldrich) to visualize the plaques; the virus titres were determined as p.f.u. $\mathrm{ml}^{-1}$.

Analysis of cytokines and chemokines. The vascular effluents from Airway Chips were collected and analysed for a panel of cytokines and chemokines-IL-6, IP10 , MCP-1, RANTES, interferon- $\beta$ and IL- 8 - using custom ProcartaPlex assay kits (Invitrogen). These cytokines were chosen because they have been previously shown to be secreted in response to influenza infection in vivo ${ }^{61}$. The analyte concentrations were determined using a Luminex 100/200 Flexmap3D instrument coupled with the Luminex XPONENT software.

Analysis of cleavage of virus HA by serine proteases. For the analysis of HA cleavage by serine proteases in the presence or absence of nafamostat, MDCK cells $\left(5 \times 10^{5}\right.$ cells per well in six-well plates) were transfected with $2.5 \mu \mathrm{g}$ serine protease expression plasmid or empty vector using the TransIT-X2 dynamic delivery system (Mirus). One day later, the cells were infected with influenza A/WSN/33 (H1N1) virus $(\mathrm{MOI}=0.01)$ in DMEM medium supplemented with $1 \%$ fetal bovine serum (FBS) and then cultured in the presence or absence of $10 \mu \mathrm{M}$ nafamostat. The supernatant was harvested two days post infection and subjected to western blot analysis using anti-HAl antibody.

Drugs for the SARS-CoV-2pp studies. Chloroquine (cat. no. ab142116), hydroxychloroquine (cat. no. ab120827), arbidol (cat. no. ab145693), toremifene (cat. no. ab142467), clomiphene (cat. no. ab141183), verapamil (cat. no. ab146680) and amiodarone (cat. no. ab141444) were purchased from Abcam; amodiaquine dihydrochloride dihydrate (cat. no. A2799) was purchased from Sigma-Aldrich; $\mathrm{N}$-desethylamodiaquine (cat. no. 20822) was purchased from Caymanchem. Chloroquine was dissolved in water to a stock concentration of $10 \mathrm{mM}$; all of the other drugs tested were dissolved in dimethyl sulfoxide to a stock concentration of $10 \mathrm{mM}$. The purity of all evaluated drugs was $>95 \%$.

Plasmids. Plasmid expressing the spike protein of SARS-CoV-2 (pCMV3-SARS-CoV2-Spike) was purchased from Sino Biological Inc. The pCMV-VSVG, pNL4-3.Luc.R-E and pAdvantage plasmids were obtained from Addgene, NIH AIDS Reagent Program and Promega, respectively. All of the plasmids used for transfection were amplified using a Maxiprep kit (Promega) according to the manufacturer's instructions.

Pseudotyped virus production. HEK293T cells $\left(5 \times 10^{5}\right.$ cells per well $)$ were seeded into six-well plates. After $24 \mathrm{~h}$, the HEK293T cells were transfected with $1.0 \mu \mathrm{g}$ pNL4-3.Luc.R-E, $0.07 \mu \mathrm{g}$ pCMV3-SARS-CoV-2-Spike and $0.3 \mu \mathrm{g}$ pAdvantage 
using the TransIT-X2 transfection reagent (Mirus) according to the manufacturer's instructions to produce SARS-CoV-2-spike-pseudotyped HIV virions (SARS-CoV 2pp). Similarly, HEK293T cells were transfected with $1.0 \mu \mathrm{g}$ pNL4-3.Luc.R-E, $0.7 \mu \mathrm{g}$ pCMV-VSVG and $0.3 \mu \mathrm{g}$ pAdvantage to produce VSVG-pseudotyped HIV virions (VSVpp). The supernatants containing the pseudotyped viruses were collected at $48 \mathrm{~h}$ post transfection and clarified by the removal of floating cells and cell debris with centrifugation at $1 \times 10^{3} \mathrm{~g}$ for $5 \mathrm{~min}$. The culture supernatants containing the pseudotyped virus particles were either used immediately or flash frozen in aliquots and stored at $-80^{\circ} \mathrm{C}$ until use after being concentrated using a PEG virus precipitation kit (Abcam). Incorporation of the SARS-CoV-2 S protein into the SARS-CoV-2pp was confirmed using western blot analysis with anti-SARS-CoV-2 S1 chimaeric monoclonal antibody with combined constant domains of the human IgG1 molecule and mouse variable regions (1:500; Sino Biological, 40150-D001); a recombinant receptor-binding-domain fragment from the $\mathrm{S} 1$ region was used as a control (BEI resources, NR-52306). Similar results were obtained using a commercially available pseudotyped SARS-CoV-2 S-protein-expressing viral particles (Amsbio LLC).

Infection assay using pseudotyped viruses in Huh-7 cells. Drugs were tested using entry assays for SARS-CoV-2pp and VSVpp as previously described ${ }^{31}$. Infections were performed in 96-well plates. SARS-CoV-2pp or VSVpp were added to $5 \times 10^{3}$ Huh-7 cells per well in the presence or absence of the test drugs or compounds. The mixtures were then incubated for $72 \mathrm{~h}$ at $37^{\circ} \mathrm{C}$. Luciferase activity, which reflects the number of pseudoparticles in the host cells, was measured at $72 \mathrm{~h}$ post infection using the Bright-Glo reagent (Promega) according to the manufacturer's instructions. The test drugs were serially diluted to a final concentration of 1 or $5 \mu \mathrm{M}$. The maximum infectivity (100\%) was derived from the untreated wells and background $(0 \%)$ from the uninfected wells. To calculate the infection values, the luciferase background signals were subtracted from the intensities measured for each of the wells exposed to the drug; this value was divided by the average signal of the untreated control wells and multiplied by $100 \%$.

SARS-CoV-2pp infection of human Airway Chips. To measure infection in human Airway Chips with the pseudotyped virus, drugs were flowed through the vascular channel of the Airway Chips at their reported $C_{\max }$ in human blood (Table 1) while the airway channel was statically treated with the same concentrations of drugs. The SARS-CoV-2pp was delivered into the airway channel $24 \mathrm{~h}$ later in a small volume $(30 \mu \mathrm{l})$ of medium containing the drug at the same concentrations and incubated statically at $37^{\circ} \mathrm{C}$ for an additional $48 \mathrm{~h}$ while the drug (at the same dose) was continuously flowed through the vascular channel. The airway epithelium was then collected using an RNeasy micro kit (Qiagen) according to the manufacturer's instructions and subjected to analysis of the viral load by RTqPCR. As we only focused in assessing viral entry in these studies, the chips were only lined by differentiated airway epithelium and did not contain endothelium.

Mass spectrometry. Human lung bronchial-airway epithelial cells cultured for four weeks at ALI were treated with $10 \mu \mathrm{M}$ amodiaquine, chloroquine or hydroxychloroquine for $48 \mathrm{~h}$. The cells were then lysed in $8 \mathrm{M}$ urea and $200 \mathrm{mM}$ EPPS pH 8.5 with protease inhibitor. The samples were prepared, labelled with tandem mass tags and analysed by liquid chromatography-tandem mass spectrometry (LC-MS/MS) on an Orbitrap Fusion Lumos mass spectrometer coupled to a Proxeon NanoLC-1200 UHPLC ${ }^{50}$. The mass spectra were processed using a Sequest-based pipeline ${ }^{16}$ and searches were performed using a 50-ppm precursor ion tolerance for the analysis of the total protein levels. The product ion tolerance was set to $0.9 \mathrm{Da}$. Tandem mass tags on lysine residues and peptide amino termini (+229.163 Da) and carbamidomethylation of cysteine residues $(+57.021 \mathrm{Da})$ were set as static modifications, whereas oxidation of methionine residues $(+15.995 \mathrm{Da})$ was set as a variable modification. Peptide-spectrum matches were adjusted to a $1 \%$ false-discovery rate. Proteins were quantified by summing the reporter-ion counts across all matching peptide-spectrum matches, as described previously ${ }^{51}$. Peptide-spectrum matches with poor quality, MS3 spectra with tandem-mass-tag reporters summed signal-to-noise of less than 100 or with no MS3 spectra were excluded from quantification. Gene Ontology analysis was performed using DAVID ${ }^{52}$; heatmaps were generated using GraphPad Prism.

Native SARS-CoV-2 in vitro infection assay. All work with native SARS-CoV-2 virus was performed in a BSL3 laboratory and approved by our Institutional Biosafety Committee. Vero E6 cells (ATCC CRL 1586) were cultured in DMEM medium (Quality Biological) supplemented with $10 \%$ (vol/vol) heat-inactivated FBS (Sigma), 1\% (vol/vol) penicillin-streptomycin (Gemini Bio-Products) and $1 \%$ (vol/vol) L-glutamine ( $2 \mathrm{mM}$ final concentration; Gibco) (Vero medium). The cells were maintained at $37^{\circ} \mathrm{C}\left(5 \% \mathrm{CO}_{2}\right)$. Native SARS-CoV-2 expressing GFP was provided by R. S. Baric ${ }^{53}$. Stocks were prepared by infection of Vero E6 cells for two days when a cytopathic effect was starting to become visible. The media were collected and clarified by centrifugation before being aliquoted for storage at $-80^{\circ} \mathrm{C}$. The titre of the stock was determined by plaque assay using Vero E6 cells. Infection and drug testing using GFP-expressing native SARS-CoV-2 were performed in Vero E6 cells. The cells were plated in clear-bottom black 96-well plates one day before infection. The drug was diluted to $50 \mu \mathrm{M}$ from the stock and an eight-point 1:2 dilution series was prepared in duplicate in Vero medium. Each drug dilution and control was normalized to contain the same concentration of drug vehicle (for example, dimethyl sulfoxide). The cells were pretreated with the drug for $2 \mathrm{~h}$ at $37^{\circ} \mathrm{C}\left(5 \% \mathrm{CO}_{2}\right)$ before infection with SARS-CoV-2 at MOI $=0.1$. The plates were then incubated at $37^{\circ} \mathrm{C}\left(5 \% \mathrm{CO}_{2}\right)$ for $48 \mathrm{~h}$, followed by fixation with $4 \%$ paraformaldehyde, nuclear staining with Hoechst (Invitrogen) and data acquisition on a Celigo five-channel imaging cytometer (Nexcelom Bioscience). The percentage of infected cells was determined for each well based on GFP expression by manual gating using the Celigo software. In addition to plates that were infected, parallel plates were left uninfected to monitor the cytotoxicity of the drug alone. The plates were incubated at $37^{\circ} \mathrm{C}\left(5 \% \mathrm{CO}_{2}\right)$ for $48 \mathrm{~h}$ before performing CellTiter-Glo (CTG) assays as per the manufacturer's instructions (Promega). Luminescence was read on a BioTek Synergy HTX plate reader using the Gen5 software (v7.07; BioTek Instruments Inc.). Similar results were obtained with wild-type SARS-CoV-2 virus, using a previously published method ${ }^{45}$.

Hamster PK studies. Amodiaquine dihydrochloride dihydrate (Sigma, cat. no. A2799) was formulated at $10 \mathrm{mg} \mathrm{ml}^{-1}$ in $12 \%$ sulfobutylether- $\beta$-cyclodextrin in water at pH 5.0 and administered to LVG male hamsters $(n=3)$ at $50 \mathrm{mg} \mathrm{kg}^{-1}$ by subcutaneous injection (dose volume of $5 \mathrm{ml} \mathrm{kg}^{-1}$ ) or in $1 \%$ methyl cellulose ( $400 \mathrm{cP}$; Sigma) in double-distilled water at $75 \mathrm{mg} \mathrm{kg}^{-1}$ by oral gavage. Blood samples were drawn at $0.5,1,2,4,8$ and $24 \mathrm{~h}$ and plasma was prepared. At $24 \mathrm{~h}$, the animals were anaesthetized and then perfused to clear the tissues of blood. The tissues of interest (lung, heart, kidney and intestine) were removed and homogenized at a ratio of 1:3 (wt/vol) in water. The desired serial concentrations of working reference analyte solutions of amodiaquine (Selleckchem) and desethylamodiaquine (Cayman Biochemicals) were achieved by diluting the stock solution of analyte with $50 \%$ acetonitrile $(0.1 \%$ formic acid) in water solution. Working solutions $(20 \mu \mathrm{l})$ were added to $20 \mu \mathrm{l}$ of the blank LVG hamster plasma to achieve calibration standards of $1-1,000 \mathrm{ng} \mathrm{ml}^{-1}$ in a total volume of $40 \mu \mathrm{l}$. The standards, quality control samples and unknown samples $(20 \mu \mathrm{l}$ plasma with $20 \mu \mathrm{l}$ blank solution) were added ( $40-\mu \mathrm{l}$ volumes) to $200 \mu \mathrm{l}$ acetonitrile containing either the internal standard or $0.1 \%$ formic acid mixture for precipitating protein. The samples were then vortexed for $30 \mathrm{~s}$. After centrifugation at 3,900 r.p.m. for $15 \mathrm{~min}$ at $4{ }^{\circ} \mathrm{C}$, the supernatant was diluted $3 \times$ with water. The diluted supernatant $(5 \mu \mathrm{l})$ was injected into the LC-MS/MS system (AB API 5500 LC-MS/MS instrument with a Phenomenex Synergi $2.5 \mu \mathrm{m}$ Polar-RP $100 \mathrm{~A}(50 \times 3 \mathrm{~mm})$ column $)$ for quantitative analysis. The mobile phases used were $95 \%$ water $(0.1 \%$ formic acid) and $95 \%$ acetonitrile ( $0.1 \%$ formic acid). All PK studies were conducted by Pharmaron and performed in accordance with the guidelines of the Institutional Animal Care and Use Committee of Pharmaron.

Hamster efficacy studies. SARS-CoV-2 isolate USA-WA1/2020 (NR-52281) was provided by the CDC. SARS-CoV-2 was propagated in Vero E6 cells in DMEM medium supplemented with $2 \% \mathrm{FBS}, 4.5 \mathrm{gl}^{-1} \mathrm{D}$-glucose, $4 \mathrm{mM} \mathrm{L}$-glutamine, $10 \mathrm{mM}$ non-essential amino acids, $1 \mathrm{mM}$ sodium pyruvate and $10 \mathrm{mM}$ HEPES, and filtered through an Amicon Ultracel $15(100 \mathrm{kDa})$ centrifugal filter. The flow-through was discarded and the virus was resuspended in DMEM supplemented as above. The infectious titres of the SARS-CoV-2 stock were determined using a plaque assay in Vero E6 cells in Minimum essential medium supplemented with $2 \%$ FBS, $4 \mathrm{mM}$ L-glutamine, $0.2 \% \mathrm{BSA}, 10 \mathrm{mM}$ HEPES, $0.12 \% \mathrm{NaHCO}_{3}$ and $0.7 \%$ agar. Syrian hamsters (3-5 weeks old) were acclimated to the CDC/USDA-approved BSL3 facility of the Global Health and Emerging Pathogens Institute at the Icahn School of Medicine at Mount Sinai for 2-4 days. In our direct infection prevention and transmission models, the hamsters were either given a subcutaneous injection posteriorly or administered an oral gavage with the drug within $2 \mathrm{~h}$ of drug reconstitution one day before infection with SARS-CoV-2, on the day of infection as well as one and two days after infection; the animals were killed and their lungs were harvested for analysis on day 3 post infection. Amodiaquine was reconstituted in $12 \%$ sulfobutylether- $\beta$-cyclodextrin (Selleckchem) in water (wt/wt; with $\mathrm{HCl}$ / $\mathrm{NaOH}$ ) at $\mathrm{pH} 5.0$ for subcutaneous injections or $1 \%$ methyl cellulose in water for oral gavage. Hydroxychloroquine was reconstituted in PBS. The hamsters were intranasally infected with $1 \times 10^{3}$ p.f.u. of passage- 3 SARS-CoV-2 USA-WA1/2020 in $100 \mu \mathrm{l}$ PBS and sacrificed on day 3 of infection. The animals were anaesthetized by intraperitoneal injection of $200 \mu \mathrm{l}$ of ketamine and xylazine (3:1) and provided with thermal support while unconscious. Whole lungs were harvested and homogenized in $1 \mathrm{ml}$ PBS and the homogenates were then spun down at 10,000 $\mathrm{g}$ for $5 \mathrm{~min}$; the supernatant was subsequently discarded and the lung pellet was resuspended in TRIzol. The same protocol was used in our animal-to-animal infection model, except amodiaquine was administered to healthy hamsters for one day before they were housed with untreated hamsters that had been infected with SARS-CoV-2 one day earlier and the drug continued to be administered daily for three more days, after which the infection transmission was quantified. In our treatment models, amodiaquine was administered via oral gavage for two days beginning on day 1 after intranasal administration of virus $\left(1 \times 10^{3}\right.$ p.f.u. $)$ and lungs were analysed at 3 or 7 days. The animal experiments were performed in accordance with the guidelines of the Institutional Animal Care and Use Committee and Institutional Biosafety Committee of the Icahn School of Medicine at Mount Sinai. 
Lung RNA was extracted by phenol-chloroform extraction and DNase treatment using a DNA-free DNA removal kit (Invitrogen). After cDNA synthesis of the RNA samples by reverse transcription using SuperScript II reverse transcriptase (Invitrogen) with oligo $\mathrm{d}(\mathrm{T})$ primers, $\mathrm{RT}-\mathrm{qPCR}$ was performed using a SYBR FAST qPCR master mix kit (Kapa Biosystems) on a LightCycler 480 instrument II (Roche) for subgenomic N RNA (sgRNA) and actin using the following primers: actin forward primer, 5' -CCAAGGCCAACCGTGAAAAG-3' actin reverse primer, $5^{\prime}$-ATGGCTACGTACATGGCTGG-3'; N sgRNA forward primer, 5' -CTCTTGTAGATCTGTTCTCTAAACGAAC-3'; and N sgRNA reverse primer, 5'-GGTCCACCAAACGTAATGCG-3'. The relative sgRNA levels were quantified by normalizing the sgRNA to actin expression and normalizing the RNA from drug-treated infected lungs to vehicle-treated infected controls. All RNA-sequencing data utilized the Illumina TruSeq stranded mRNA LP as per the manufacturer's instructions. Illumina libraries were quantified using Qubit and an Agilent Bioanalyzer before being run on an Illumina NextSeq500 using a high-capacity flow cell. All raw data were processed as described elsewhere ${ }^{62}$. The sequencing datasets generated during this study are available on the NCBI Gene Expression Omnibus (GEO) server under the accession number GSE171711. The sequencing datasets for DMSO treated SARS-CoV-2 infected A549-ACE2 cells can be found on the NCBI GEO server under the accession number GSE159522 (GSM4831772, GSM4831773 and GSM4831774).

Statistical analysis. All of the results presented are from at least two independent experiments and all of the data points shown indicate the mean \pm s.d. from multiple biological replicates or Organ Chips. The Airway Chips used in these studies were created with cells obtained from more than five different donors and produced consistent results. Tests for statistically significant differences between groups were performed using a two-tailed Student's $t$-test and the Bonferroni correction for multiple hypothesis testing, and all $P$ values are presented in the figures unless $P<0.001$, which is then indicated in the legend.

Reporting Summary. Further information on research design is available in the Nature Research Reporting Summary linked to this article.

\section{Data availability}

The nucleotide sequences used in the study have been deposited in GeneBank under the accession numbers CY034139.1, CY0334138.1, X17336.1, HE802059.1, CY034135.1, CY034134.1, D10598.1, M12597.1, CY176949.1, CY176948.1, CY176947.1, CY176942.1, CY176945.1, CY176944.1, CY176943.1, CY176946.1, DQ487334.1, DQ487333.1, DQ487335.1, DQ487340.1, DQ487339.1, DQ487337.1, DQ487338.1 and DQ487336.1. The raw sequencing data files generated in the experiments with hamsters can be found on NCBI Gene Expression Omnibus (GSE143613). All data generated or analysed during this study are available from the corresponding author on reasonable request.

Received: 31 December 2020; Accepted: 19 March 2021; Published online: 3 May 2021

\section{References}

1. Boulware, D. R. et al. A randomized trial of hydroxychloroquine as postexposure prophylaxis for COVID-19. N. Engl. J. Med. 383, 517-525 (2020)

2. Borba, M. G. S. et al. Effect of high vs low doses of chloroquine diphosphate as adjunctive therapy for patients hospitalized with severe acute respiratory syndrome coronavirus 2 (SARS-CoV-2) infection: a randomized clinical trial. JAMA Netw. Open 3, e208857 (2020).

3. Hu, T. Y., Frieman, M. \& Wolfram, J. Insights from nanomedicine into chloroquine efficacy against COVID-19. Nat. Nanotechnol. 15 247-249 (2020).

4. Funnel, E. A. Emerging preclinical evidence does not support broad use of hydroxychloroquine in COVID-19 patients. Nat. Commun. 11, 4253 (2020)

5. Huh, D. et al. Reconstituting organ-level lung functions on a chip. Science 328, 1662-1668 (2010)

6. Huh, D. et al. A human disease model of drug toxicity-induced pulmonary edema in a lung-on-a-chip microdevice. Sci. Transl. Med. 4, 159 ral 147 (2012)

7. Chou, D. B. et al. On-chip recapitulation of clinical bone marrow toxicities and patient-specific pathophysiology. Nat. Biomed. Eng. 4, 394-406 (2020).

8. Herland, A. et al. Quantitative prediction of human pharmacokinetic responses to drugs via fluidically coupled vascularized organ chips. Nat. Biomed. Eng. 4, 421-436 (2020).

9. Prantil-Baun, R. et al. Physiologically based pharmacokinetic and pharmacodynamic analysis enabled by microfluidically linked Organs-on-Chips. Annu. Rev. Pharmacol. Toxicol. 58, 37-64 (2018)

10. Hui, K. P. Y. et al. Tropism, replication competence, and innate immune responses of influenza virus: an analysis of human airway organoids and ex-vivo bronchus cultures. Lancet Respir. Med. 6, 846-854 (2018).

11. Ramani, S., Crawford, S. E., Blutt, S. E. \& Estes, M. K. Human organoid cultures: transformative new tools for human virus studies. Curr. Opin. Virol. 29, 79-86 (2018).
12. Chan, R. W., Chan, M. C., Nicholls, J. M. \& Malik Peiris, J. S. Use of ex vivo and in vitro cultures of the human respiratory tract to study the tropism and host responses of highly pathogenic avian influenza A (H5N1) and other influenza viruses. Virus Res. 178, 133-145 (2013).

13. Takayama, K. In vitro and animal models for SARS-CoV-2 research. Trends Pharmacol. Sci. 41, 513-517 (2020).

14. Stojdl, D. F. et al. VSV strains with defects in their ability to shutdown innate immunity are potent systemic anti-cancer agents. Cancer Cell 4, 263-275 (2003).

15. Ainslie, G. R. et al. Microphysiological lung models to evaluate the safety of new pharmaceutical modalities: a biopharmaceutical perspective. Lab Chip https://doi.org/10.1039/c9lc00492k (2019).

16. Ingber, D. E. Is it time for Reviewer 3 to request human Organ Chip experiments instead of animal validation studies? Adv. Sci. https://doi org/10.1002/advs.202002030 (2020).

17. Benam, K. H. et al. Small airway-on-a-chip enables analysis of human lung inflammation and drug responses in vitro. Nat. Methods $\mathbf{1 3}$ 151-157 (2016)

18. Yaghi, A. \& Dolovich, M. B. Airway epithelial cell cilia and obstructive lung disease. Cells https://doi.org/10.3390/cells5040040 (2016).

19. Jia, H. Pulmonary angiotensin-converting enzyme 2 (ACE2) and inflammatory lung disease. Shock 46, 239-248 (2016).

20. Hoffmann, M. et al. SARS-CoV-2 cell entry depends on ACE2 and TMPRSS2 and is blocked by a clinically proven protease inhibitor. Cell $\mathbf{1 8 1}$ 271-280 (2020).

21. Sungnak, W. et al. SARS-CoV-2 entry factors are highly expressed in nasal epithelial cells together with innate immune genes. Nat. Med. 26 681-687 (2020)

22. Armstrong, S. M., Mubareka, S. \& Lee, W. L. The lung microvascular endothelium as a therapeutic target in severe influenza. Antivir. Res. 99, 113-118 (2013).

23. van Riel, D. et al. H5N1 virus attachment to lower respiratory tract. Science 312, 399 (2006).

24. Kwok, K. O. et al. Relative incidence and individual-level severity of seasonal influenza A H3N2 compared with 2009 pandemic H1N1. BMC Infect. Dis. 17, 337 (2017).

25. Papayannopoulos, V. Neutrophil extracellular traps in immunity and disease. Nat. Rev. Immunol. 18, 134-147 (2018).

26. Cheung, C. Y. et al. Induction of proinflammatory cytokines in human macrophages by influenza A (H5N1) viruses: a mechanism for the unusual severity of human disease? Lancet 360, 1831-1837 (2002).

27. Nicholson, K. G. et al. Efficacy and safety of oseltamivir in treatment of acute influenza: a randomised controlled trial. Neuraminidase Inhibitor Flu Treatment Investigator Group. Lancet 355, 1845-1850 (2000).

28. Zhou, J. et al. Differentiated human airway organoids to assess infectivity of emerging influenza virus. Proc. Natl Acad. Sci. USA 115, 6822-6827 (2018).

29. Laporte, M. \& Naesens, L. Airway proteases: an emerging drug target for influenza and other respiratory virus infections. Curr. Opin. Virol. 24, 16-24 (2017).

30. Simonson, W. Influenza treatment with antiviral medications. Geriatr. Nurs. 40, 99-100 (2019).

31. Si, L. et al. Triterpenoids manipulate a broad range of virus-host fusion via wrapping the HR2 domain prevalent in viral envelopes. Sci. Adv. 4, eaau8408 (2018)

32. Wu, F. et al. A new coronavirus associated with human respiratory disease in China. Nature 579, 265-269 (2020).

33. Ou, X. et al. Characterization of spike glycoprotein of SARS-CoV-2 on virus entry and its immune cross-reactivity with SARS-CoV. Nat. Commun. 11, 1620 (2020).

34. Letko, M., Marzi, A. \& Munster, V. Functional assessment of cell entry and receptor usage for SARS-CoV-2 and other lineage B betacoronaviruses. Nat. Microbiol. 5, 562-569 (2020).

35. Johansen, L. M. et al. FDA-approved selective estrogen receptor modulators inhibit Ebola virus infection. Sci. Transl. Med. 5, 190 ra179 (2013).

36. Johansen, L. M. et al. A screen of approved drugs and molecular probes identifies therapeutics with anti-Ebola virus activity. Sci. Transl. Med. 7, $290 \mathrm{ra} 289$ (2015)

37. Zhao, Y. et al. Toremifene interacts with and destabilizes the Ebola virus glycoprotein. Nature 535, 169-172 (2016).

38. Hattermann, K. et al. Susceptibility of different eukaryotic cell lines to SARS-coronavirus. Arch. Virol. 150, 1023-1031 (2005).

39. Matsuyama, S. et al. Enhanced isolation of SARS-CoV-2 by TMPRSS2-expressing cells. Proc. Natl Acad. Sci. USA 117, 7001-7003 (2020).

40. Chen, J. et al. Efficacies of lopinavir/ritonavir and abidol in the treatment of novel coronavirus pneumonia (in Chinese). Chin. J. Infect. Dis. 38, E008-E008 (2020)

41. Orrell, C. et al. Pharmacokinetics and tolerability of artesunate and amodiaquine alone and in combination in healthy volunteers. Eur. J. Clin. Pharm. 64, 683-690 (2008).

42. Gendrot, M. et al. Antimalarial drugs inhibit the replication of SARS-CoV-2: an in vitro evaluation. Travel Med. Infect. Dis. 37, 101873 (2020). 
43. Bocci, G. et al. Virtual and in vitro antiviral screening revive therapeutic drugs for COVID-19. ACS Pharm. Transl. Sci. 3, 1278-1292 (2020).

44. Imai, M. et al. Syrian hamsters as a small animal model for SARS-CoV-2 infection and countermeasure development. Proc. Natl Acad. Sci. USA 117, 16587-16595 (2020).

45. Osterrieder, N. et al. Age-dependent progression of SARS-CoV-2 Infection in Syrian Hamsters. Viruses https://doi.org/10.3390/v12070779 (2020).

46. Hoagland, D. A. et al. Leveraging the antiviral type I interferon system as a first line of defense against SARS-CoV-2 pathogenicity. Immunity https:/doi. org/10.1016/j.immuni.2021.01.017 (2021).

47. $\mathrm{Si}$, L. et al. Human Organ Chip-enabled pipeline to rapidly repurpose therapeutics during viral pandemics. Preprint at bioRxiv https://doi. org/10.1101/2020.04.13.039917 (2020).

48. Deinhardt-Emmer, S. et al. SARS-CoV-2 causes severe alveolar inflammation and barrier dysfunction. J. Virol. https://doi.org/10.1128/JVI.00110-21 (2021).

49. Thacker, V. V. et al. Rapid endothelialitis and vascular inflammation characterise SARS-CoV-2 infection in a human lung-on-chip model. Preprint at bioRxiv https://doi.org/10.1101/2020.08.10.243220 (2020).

50. Zhang, M. et al. Biomimetic human disease model of SARS-CoV-2 induced lung injury and immune responses on Organ Chip system. Adv. Sci. https://doi.org/10.1002/advs.202002928 (2020).

51. Marx, U. et al. Biology-inspired microphysiological systems to advance patient benefit and animal welfare in drug development. ALTEX https://doi. org/10.14573/altex.2001241 (2020).

52. Tang, B. M. et al. Neutrophils-related host factors associated with severe disease and fatality in patients with influenza infection. Nat. Commun. 10, 3422 (2019).

53. de Oliveira, S., Rosowski, E. E. \& Huttenlocher, A. Neutrophil migration in infection and wound repair: going forward in reverse. Nat. Rev. Immunol. 16, 378-391 (2016).

54. Li, Y. et al. Efficacy and safety of lopinavir/ritonavir or arbidol in adult patients with mild/moderate COVID-19: an exploratory randomized controlled trial. Med https://doi.org/10.1016/j.medj.2020.04.001 (2020).

55. Jeon, S. et al. Identification of antiviral drug candidates against SARS-CoV-2 from FDA-approved drugs. Antimicrob. Agents Chemother. https://doi. org/10.1128/AAC.00819-20 (2020).

56. Weston, S. et al. Broad anti-coronavirus activity of Food and Drug Administration-approved drugs against SARS-CoV-2 in vitro and SARS-CoV in vivo. J. Virol. https://doi.org/10.1128/JVI.01218-20 (2020).

57. DeWald, L. E. et al. In vivo activity of amodiaquine against Ebola virus infection. Sci. Rep. 9, 20199 (2019).

58. Sinou, V. et al. Pharmacokinetics and pharmacodynamics of a new ACT formulation: artesunate/amodiaquine (TRIMALACT) following oral administration in African malaria patients. Eur. J. Drug Metab. Pharmacokinet. 34, 133-142 (2009)

59. Gignoux, E. et al. Effect of artesunate-amodiaquine on mortality related to Ebola virus disease. N. Engl. J. Med. 374, 23-32 (2016).

60. CDC International notes agranulocytosis associated with the use of amodiaquine for malaria prophylaxis. MMWR 35, 165-166 (1986).

61. Short, K. R., Kroeze, E., Fouchier, R. A. M. \& Kuiken, T. Pathogenesis of influenza-induced acute respiratory distress syndrome. Lancet Infect. Dis. 14, 57-69 (2014).
62. Blanco-Melo, D. et al. Imbalanced host response to SARS-CoV-2 drives development of COVID-19. Cell 181, 1036-1045 (2020).

\section{Acknowledgements}

We thank the CDC, A. Garcia-Sastre, R. A. M. Fouchier and X. Sealens for providing the influenza virus strains and the influenza virus rescue systems. We acknowledge research funding from the NIH (grant nos NCATS 1-UG3-HL-141797-01 and NCATS 1-UH3-HL-141797-01 to D.E.I.), DARPA under Cooperative Agreements (grant nos W911NF-12-2-0036 to D.E.I. and W911NF-16-C-0050 to D.E.I., M.F. and B.R.t.), the Bill and Melinda Gates Foundation (to D.E.I. and M.F.), the Marc Haas Foundation (to B.R.t.) and the Wyss Institute for Biologically Inspired Engineering at Harvard University (to D.E.I.).

\section{Author contributions}

L.S., H.B. and D.E.I. conceived this study, and D.E.I. developed the overall collaborative discovery pipeline. L.S. and H.B. performed and analysed experiments with other authors assisting with experiments and data analysis. M.R. assisted with the cytokine detection assay. W.C., C.O., A.J., A.N. and S.M.K. assisted with RNA extraction and RT-qPCR. D.Y.Z. and G.G. assisted in the characterization of CoV-2pp. S.P.G. assisted in the mass spectrometry experiments. R.K.P. assisted with the statistical analysis. R.P. and S.E.G. coordinated the experiments and managed the project progress. R.M., D.H., K.O., R.A.A., W.-C.L., S.H., T.J., J.F., I.G. and B.R.t. developed the hamster COVID-19 infection model and tested the efficacy of drugs against native SARS-CoV-2 in vivo. K.E.C. coordinated the hamster PK studies and assisted in the design of dosing and drug formulation in the hamster efficacy studies. J.L., R.H., M.M., S.W. and M.F. tested the activity of amodiaquine and desethylamodiaquine against native SARS-CoV-2 in Vero E6 cells. L.S., H.B. and D.E.I. wrote the manuscript with all authors providing feedback.

\section{Competing interests}

D.E.I. is a founder, board member and Scientific Advisory Board chair of, and holds equity in, Emulate Inc. D.E.I., L.S., R.P., H.B., K.H.B. and M.R. are inventors on the relevant patent applications held by Harvard University (patents PCT/US2020/030697, PCT/US2020/033590, PCT/US2020/024556, 62-987-024, 62-826-45 and 62850113). The remaining authors declare no competing interests.

\section{Additional information}

Supplementary information The online version contains supplementary material available at https://doi.org/10.1038/s41551-021-00718-9.

Correspondence and requests for materials should be addressed to D.E.I.

Peer review information Nature Biomedical Engineering thanks the anonymous reviewers for their contribution to the peer review of this work. Peer reviewer reports are available.

Reprints and permissions information is available at www.nature.com/reprints.

Publisher's note Springer Nature remains neutral with regard to jurisdictional claims in published maps and institutional affiliations.

(c) The Author(s), under exclusive licence to Springer Nature Limited 2021 


\section{Reporting Summary}

Nature Research wishes to improve the reproducibility of the work that we publish. This form provides structure for consistency and transparency in reporting. For further information on Nature Research policies, see our Editorial Policies and the Editorial Policy Checklist.

\section{Statistics}

For all statistical analyses, confirm that the following items are present in the figure legend, table legend, main text, or Methods section.

n/a Confirmed

$\bigotimes$ The exact sample size $(n)$ for each experimental group/condition, given as a discrete number and unit of measurement

$\searrow$ A statement on whether measurements were taken from distinct samples or whether the same sample was measured repeatedly

The statistical test(s) used AND whether they are one- or two-sided

Only common tests should be described solely by name; describe more complex techniques in the Methods section.

Х $\square$ A description of all covariates tested

$\bigotimes$ A description of any assumptions or corrections, such as tests of normality and adjustment for multiple comparisons

A full description of the statistical parameters including central tendency (e.g. means) or other basic estimates (e.g. regression coefficient)

AND variation (e.g. standard deviation) or associated estimates of uncertainty (e.g. confidence intervals)

For null hypothesis testing, the test statistic (e.g. $F, t, r$ ) with confidence intervals, effect sizes, degrees of freedom and $P$ value noted

Give $P$ values as exact values whenever suitable.

Х $\square$ For Bayesian analysis, information on the choice of priors and Markov chain Monte Carlo settings

Х $\square$ For hierarchical and complex designs, identification of the appropriate level for tests and full reporting of outcomes

Х $\square$ Estimates of effect sizes (e.g. Cohen's $d$, Pearson's $r$ ), indicating how they were calculated

Our web collection on statistics for biologists contains articles on many of the points above.

\section{Software and code}

Policy information about availability of computer code Data collection Images were taken with the built-in software of a confocal laser-scanning microscope (SP5 X MP DMI-6000, Germany). Image processing was
done using Imaris (Bitplane, Switzerland). Luminescence was read on a BioTek Synergy HTX plate reader (BioTek Instruments Inc., Winooski, VT) using the Gen5 software (v7.07, Biotek Instruments Inc., Winooski, VT). Cytokines and chemokines were determined using a Luminex100/200 Flexmap3D instrument coupled with Luminex XPONENT software (Luminex, USA). qPCR experiments were conducted using CFX96 Touch Real-Time PCR Detection System (Bio-Rad).

Data analysis Graphpad Prism 8.0.1.244, Microsoft Excel v16.

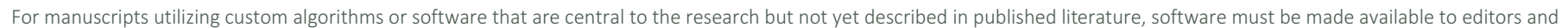

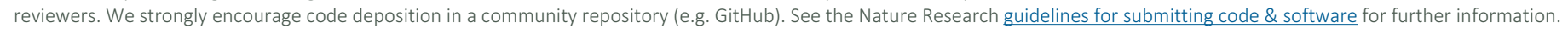

\section{Data}

Policy information about availability of data

All manuscripts must include a data availability statement. This statement should provide the following information, where applicable:

- Accession codes, unique identifiers, or web links for publicly available datasets

- A list of figures that have associated raw data

- A description of any restrictions on data availability

The nucleotide sequences used in the study have been deposited in GeneBank under accession numbers CY034139.1, CY0334138.1, X17336.1, HE802059.1, CY034135.1, CY034134.1, D10598.1, M12597.1, CY176949.1, CY176948.1, CY176947.1, CY176942.1, CY176945.1, CY176944.1, CY176943.1, CY176946.1, DQ487334.1, DQ487333.1, DQ487335.1, DQ487340.1, DQ487339.1, DQ487337.1, DQ487338.1 and DQ487336.1. The raw sequencing data files generated in the 


\section{Field-specific reporting}

Please select the one below that is the best fit for your research. If you are not sure, read the appropriate sections before making your selection. \Life sciences Behavioural \& social sciences Ecological, evolutionary \& environmental sciences

For a reference copy of the document with all sections, see nature.com/documents/nr-reporting-summary-flat.pdf

\section{Life sciences study design}

All studies must disclose on these points even when the disclosure is negative.

Sample size $\quad$ For the cell-line studies with Huh-7, Vero E6 and ACE2-expressing A549, at least 3 independent experiments were performed. For the human-lung-chip studies, at least 3 chips per donor were used and at least five donors were used. In order to increase the statistical reliability for efficacy of tested drugs, up to 6 chips were used.

For PK studies conducted in hamsters, 3 hamsters were used for the amodiaquine PK study, with blood samples taken at the indicated time points.

For the in vivo study in COVID-19 hamsters, all experiments were performed in triplicate, with six hamsters per cohort per experiment (a total of 18 hamsters treated with vehicle or amodiaquine and infected with SARS-CoV-2). All animals were treated subcutaneously. Amodiaquine administration was performed in a solution of $12 \%$ SB-beta-cyclodextrin, and administered at a concentration of $50 \mathrm{mg} / \mathrm{kg}$. Vehicle control animals were treated with only $12 \%$ SB-beta-cyclodextrin. Treatment with vehicle or amodiaquine was performed one day prior to infection, at the day of infection, and at days two and three of infection. Infection of SARS-CoV- 2 was performed intranasally (1,000 plaque-forming units) under general anesthesia (xylene and ketamine).

Data exclusions No data were excluded from the analyses.

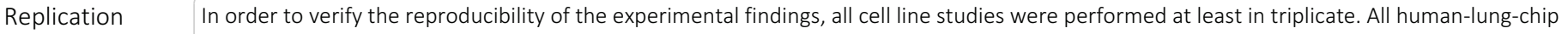
studies were performed at least in duplicate. The PK study and the in vivo COVID-19 study in hamsters were performed at least in triplicate, within the institutional Biosafetly Level 3 facility. All the attempts were successful and no data were excluded from the analyses.

Randomization The position of each drug in the plates was randomly chosen for both the primary screen and the further validations. The human lung chips were randomly assigned into groups in the human-lung-chip-model studies. Hamsters were randomly assigned into groups in all animal-model studies.

Blinding Data collection and analysis for cytokines and chemokines induced by virus infection in human lung-airway chips, and gene levels detected by RT-qPCR, were run blinded, with the investigators only having number IDs of samples (and thus not knowing their identity). Data collection and analysis for the primary screen and the validation in Huh-7 cells were run blinded, without knowing which drug was spot in the specific wells. Blinding was used for the data analysis of the SARS-CoV-2 infection and treatment study in hamsters (including the samples given to animals for treatment). Blinding was not required for the other experiments presented because the experiments were highly quantitative and controlled. Blinding was not conducted for the PK studies since the researchers had to be aware of the drug used to know what the analytes were in order to measure them by mass spectrometry.

\section{Reporting for specific materials, systems and methods}

We require information from authors about some types of materials, experimental systems and methods used in many studies. Here, indicate whether each material, system or method listed is relevant to your study. If you are not sure if a list item applies to your research, read the appropriate section before selecting a response.

\section{Materials \& experimental systems}

n/a Involved in the study

$\bigotimes$ Antibodies

$\bigotimes$ Eukaryotic cell lines

Х $\square$ Palaeontology and archaeology

$\square$ Animals and other organisms

$\bigotimes \square$ Human research participants

\ $\square$ Clinical data

$\bigotimes \square$ Dual use research of concern

\author{
Methods \\ $\mathrm{n} / \mathrm{a}$ Involved in the study \\ X $\square$ ChIP-seq \\ Х $\square$ Flow cytometry \\ Х $\square$ MRI-based neuroimaging
}

\section{Antibodies}

Antibodies used Primary antibodies included Alexa Fluor 594 labeled antibody against ZO-1 (Life Technologies; Cat\# 339194), Alexa Fluor 647 labeled 

Cat\# 560411), antibody against Mucin5Ac (Santa Cruz Biotechnology; Cat\# sc-21701), antibody against human Uteroglobin/cc-10 (R\&D Systems; Cat\# MAB4218SP), antibody against Cytokeratin 5 (Sigma-Aldrich; Cat\# SAB5300265), antibody against influenza NP (Invitrogen; Cat\# MA516291), antibody against Collagen IV a1 (Novus Biologicals; Cat\# NBP1-97716G), Alexa Fluor 594 labeled antibody against human CD45 (Biolegend, Cat\# 368520), antibody against influenza H1N1 HA1 (Sino Biological; Cat\# 11692-T62), antibody against influenza H3N2 HA1 (Abcam; Cat\# ab139361), Anti-hACE2 antibody (Abcam; cat. \#ab239924), SARS-CoV-2 (2019nCoV) Spike RBD Antibody (Sino Biological; Cat\# 40592-T62), mouse anti-TMPRSS2 (Novus Biologicals; Cat\# H00007113-B01P), rabbit anti-TMPRSS4 (Novus Biologicals; Cat\# NBP1-56991), mouse anti-TMPRSS211D (Abnova; Cat\# H00009407-B01), and rabbit antiTMPRSS11E (OriGene; Cat\# TA350522). Secondary antibodies included Goat anti-mouse IgG conjugated with Alexa Fluor 488/594/647 (Life Technologies), Goat anti-rabbit IgG conjugated with Alexa Fluor 488/594/647 (Life Technologies), Goat anti-rabbit IgG H\&L (HRP) (Abcam), and Goat anti-mouse IgG H\&L (HRP) (Abcam). In staining experiments, all primary antibodies were used in 1:100 dilution. In western blotting experiments, all primary antibodies were used in 1:2,000 dilution. Secondary antibodies were used in 1:2000 dilution. Note that we only provided catalog numbers for these antibodies, because the antibodies used in this study has very stable performance across different lots in our experience. All the antibodies have also been listed in the Supplementary Table 2.

Validation

All antibodies were obtained from commercial vendors. We relied on information provided in the corresponding Data Sheets provided by the manufacturers.

\section{Eukaryotic cell lines}

Policy information about cell lines

Cell line source(s)

Human Embryonic Kidney 293T cell line (ATCC CRL-3216), MDCK cell line (ATCC CRL-2936), and Vero E6 cells (ATCC\# CRL 1586) were obtained from the American Type Culture Collection (ATCC). The Huh-7 cell line (JCRB0403) was obtained from JCRB Cell Bank. The A549 human lung epithelial cell line was purchased from ATCC (ATCC CCL-185), and was maintained in $\mathrm{DMEM}+10 \% \mathrm{FBS}$ and transduced with a lentivirus expressing puromycin resistance and the open reading frame of the human ACE2 transcript. Cells were selected with puromycin at a concentration of 5 micrograms per milliliter. Individual clones were isolated and grown out and individually checked for ACE2 expression by western blot. Primary human lung airway epithelial cells (\#448571, \#446317, \#623950, \#485960, \#672447, \#370751) were purchased from Lonza, USA. Primary human pulmonary microvascular endothelial cells (\#H-6011) were purchased from Cell Biologics, USA. Neutrophils were isolated from human blood provided by Research Blood Components, LLC.

Authentication

The HEK 293T, MDCK, Vero E6, and Huh-7 cell lines were cultured according to the instructions of ATCC and JCRB Cell Bank, respectively. ACE2-expressing A549 cells were validated for ACE2 expression by western blot. The primary human lung airway cells were cultured using airway epithelial-cell growth medium (Promocell, Germany) according to the manufacturer's instructions. The primary human pulmonary microvascular endothelial cells were cultured using human endothelial-cell growth medium (Cell Biologics, USA) according to the manufacturer's instructions. All cells showed expected cell morphology, growth behaviour, and cell type-specific responses. No further authentication was performed.

Mycoplasma contamination

All cells were examined, and found to be negative for mycoplasma contamination.

Commonly misidentified lines

(See ICLAC register)

No commonly misidentified cell lines were used in this study.

\section{Animals and other organisms}

Policy information about studies involving animals; ARRIVE guidelines recommended for reporting animal research

Laboratory animals In the PK study, the hamsters used were 6-8 week old, male LVG Golden Syrian Hamsters. For the in vivo SARS-CoV-2 infection and treatment studies, we used outbred male Syrian golden hamsters, 3-5 weeks of age, purchased from Charles River Laboratories.

Wild animals The study did not involve wild animals.

Field-collected samples The study did not involve samples collected from the field.

Ethics oversight Ethics oversight for the hamster PK study was provided by the Institutional Animal Care and Use Committee (IACUC) at the CRO, Pharmaron (protocol number, PK-HAM-06012020, approved 26/03/2020; attending veterinarian, Wei Liu).

For the in vivo SARS-CoV-2 infection and treatment studies, all animal experiments were performed according to protocols approved by the IACUC and the Institutional Biosafety Committee of the Icahn School of Medicine at Mount Sinai (ISMMS). The animal experiments were performed in CDC-USDA-approved BSL-3 facility of the Global Health and Emerging Pathogens Institute at the Icahn School of Medicine at Mount Sinai. 